\title{
Catalyst- and Solvent-free Synthesis of Imidazo[1,2-a]pyridines
}

\author{
Dong-Jian Zhu, ${ }^{a}$ Jiu-Xi Chen, ${ }^{*, a}$ Miao-Chang Liu, ${ }^{a}$ Jin-Chang Ding ${ }^{a, b}$ and Hua-Yue Wu ${ }^{*, a}$ \\ ${ }^{a}$ College of Chemistry and Materials Engineering, Wenzhou University, Wenzhou, 325027, China \\ ${ }^{b}$ Wenzhou Vocational and Technical College, Wenzhou, 325035, China
}

\begin{abstract}
Um método altamente eficiente e simples foi descrito para a síntese de imidazo[1,2- $a$ ]piridinas pela condensação de $\alpha$-halocetonas ( $\mathrm{ArCOCHXR}{ }^{2}, \mathrm{Ar}=\mathrm{C}_{6} \mathrm{H}_{5}, 4-\mathrm{MeOC}_{6} \mathrm{H}_{4}, 4-\mathrm{ClC}_{6} \mathrm{H}_{4}, 2,4-\mathrm{Cl}_{2} \mathrm{C}_{6} \mathrm{H}_{3}$; $\mathrm{X}=\mathrm{Br}, \mathrm{Cl} ; \mathrm{R}^{2}=\mathrm{H}, \mathrm{CH}_{3}$ ) com 2-aminopiridinas, apresentando rendimentos entre bons a excelentes sem a necessidade de adição de catalisador e solvente.
\end{abstract}

A highly efficient and facile method has been described for the synthesis of imidazo[1,2- $a$ ] pyridines in good to excellent yields by condensation of the $\alpha$-haloketones $\left(\mathrm{ArCOCHXR}^{2}, \mathrm{Ar}=\right.$ $\mathrm{C}_{6} \mathrm{H}_{5}, 4-\mathrm{MeOC}_{6} \mathrm{H}_{4}, 4-\mathrm{ClC}_{6} \mathrm{H}_{4}, 2,4-\mathrm{Cl}_{2} \mathrm{C}_{6} \mathrm{H}_{3} ; \mathrm{X}=\mathrm{Br}, \mathrm{Cl} ; \mathrm{R}^{2}=\mathrm{H}, \mathrm{CH}_{3}$ ) with 2-aminopyridines without the use of any additional catalyst and solvent.

Keywords: imidazo[1,2-a]pyridines, $\alpha$-haloketones, 2-aminopyridines, catalyst-free, solvent-free

\section{Introduction}

It has long been known that imidazo[1,2- $a]$ pyridine derivatives exhibit diverse biological activities ${ }^{1}$ and were used as antiviral, ${ }^{2}$ antiulcer, ${ }^{3}$ antibacterial, ${ }^{4}$ antifungal, ${ }^{5}$ antiprotozoal, ${ }^{6}$ antiherpes, ${ }^{7}$ anti-inflammatory. ${ }^{8}$ Recently, Leopoldo et al. ${ }^{9}$ reported synthetic approaches leading to

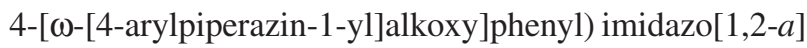
pyridine derivatives (Figure 1, I), which were described as fluorescent high-affinity dopamine $\mathrm{D}_{3}$ receptor ligands as potential probes for receptor visualization, and the fluorescent moiety compound 2-(4-methoxyphenyl) imidazo[1,2- $a$ ]pyridine (Figure 1, II), which is characterized by the 2-phenylimidazo[1,2- $a$ ]pyridine moiety, presented an oxygen that can be easily functionalized to afford potential $\mathrm{D}_{3}$ receptor ligands structurally related to the $\mathrm{D}_{3}$ receptor ligands. ${ }^{10}$ The majority of reported imidazo[1,2-a]pyridines syntheses proceeded from the condensation reaction of the $\alpha$-bromocarbonyl compounds with 2-aminopyridine derivative under neutral ${ }^{11}$ or weak basic conditions. ${ }^{12}$ A mechanism for the reaction has been proposed, ${ }^{13}$ which includes the nucleophilic substitution of the bromide by the pyridine-nitrogen in the 2-aminopyridine derivative. Imidazo[1,2-a]pyridine derivatives were also synthesized by solid support ${ }^{14}$ and using catalyst such

*e-mail: jiuxichen@wzu.edu.cn; huayuewu@wzu.edu.cn as $\mathrm{Al}_{2} \mathrm{O}_{3}{ }^{15}$ and $\mathrm{TiCl}_{4} \cdot{ }^{16}$ Other methodologies included treating 2-aminopyridines with $\alpha$-tosyloxyketones, ${ }^{17} \mathrm{a}$ polymer supported [hydroxy(sulfonyloxy)iodo]benzene with ketones or alcohols, ${ }^{18}$ alkynyl(phenyl)iodonium salts, ${ }^{19} \alpha$-diazoketones, ${ }^{20}$ and propargyl bromide. ${ }^{21}$ Although these methods are suitable for certain synthetic conditions sometimes, however, some of these procedures are associated with one or more disadvantages such as hazardous organic solvents, high cost, long reaction time, low yield, use of stoichiometric and even excess amounts of reagents or catalysts, special apparatus and drastic reaction conditions, which leaves scope for further development of new environmentally clean syntheses.
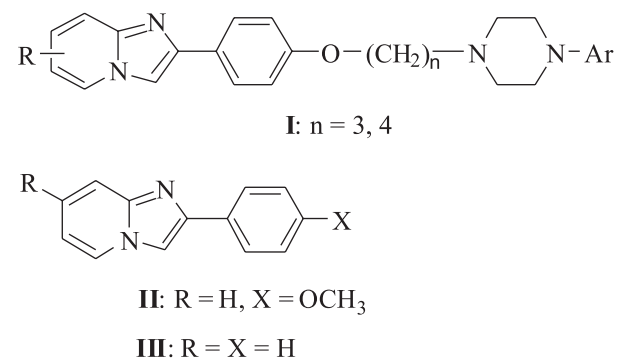

Figure 1.

As the increase in environmental consciousness chemical research and industry, ${ }^{22}$ the challenge for a sustainable environment calls for clean procedures that can avoid using harmful organic solvents, or even better, do not 
need solvent at all. In continuation of our interest in green chemistry, ${ }^{23}$ we herein wish to report the neat reaction of 2 -aminopyridine with $\alpha$-haloketones under catalyst- and solvent-free conditions, affording imidazo[1,2- $a]$ pyridine derivatives in good to excellent yields.

\section{Results and Discussion}

To optimize the reaction conditions, initial studies were concentrated on reactions of the $\alpha$-bromoacetophenone with 2-aminopyridine as a model reaction. After careful screening, to our delight, the reaction occurred for $20 \mathrm{~min}$ affording 2-phenylimidazo[1,2-a]pyridine (3a) in $91 \%$ yield at $60{ }^{\circ} \mathrm{C}$ in the absence of catalyst and solvent (Table 1, entry 11). It was observed that the mixture was initially in a solid state, and then turned to liquid state during the process of stirring, finally solidified to a light yellow solid mass.

Table 1. The condensation of 2-aminopyridine with $\alpha$-bromoacetophenone under different reaction conditions ${ }^{\mathrm{a}}$

\begin{tabular}{lccc}
\hline Entry & Solvent & $\mathrm{T} /\left({ }^{\circ} \mathrm{C}\right)$ & Yield / $(\%)^{\mathrm{b}}$ \\
\hline 1 & $n$-Hexane & 60 & 48 \\
2 & $\mathrm{CCl}_{4}$ & 60 & 45 \\
3 & $\mathrm{Toluene}_{2}$ & 60 & 50 \\
4 & $\mathrm{CH}_{2} \mathrm{Cl}_{2}$ & 60 & 53 \\
5 & $\mathrm{THF}_{3}$ & 60 & 65 \\
6 & $\mathrm{CH}_{3} \mathrm{CN}$ & 60 & 59 \\
7 & $\mathrm{C}_{2} \mathrm{H}_{5} \mathrm{OH}$ & 60 & 60 \\
8 & $\mathrm{CH}_{3} \mathrm{OH}$ & 60 & 63 \\
9 & $\mathrm{PEG}^{2}-400$ & 60 & 60 \\
10 & $\mathrm{H}_{2} \mathrm{O}$ & 60 & 63 \\
11 & none & 60 & 91 \\
12 & none & 25 & $65^{\mathrm{c}}$ \\
\hline
\end{tabular}

a Reaction conditions: $\alpha$-bromoacetophenone $(1.0 \mathrm{mmol}), 2$-aminopyridine $(1.0 \mathrm{mmol})$, solvent $(1 \mathrm{~mL})$ at $60{ }^{\circ} \mathrm{C}$ for $20 \mathrm{~min}$. 'bsolated yield. ${ }^{\mathrm{c}} \mathrm{The}$ reaction was carried out at room temperature for $24 \mathrm{~h}$.

Encouraged by this result, we further carried out comparative reactions to optimize the reaction conditions. The results are summarized in Table 1. When the reaction was carried out at room temperature in the absence of catalyst and solvent, only $65 \%$ yield was obtained, even when the reaction time was prolonged to $24 \mathrm{~h}$ (Table 1, entry 12). The nonpolar solvents, such as $n$-hexane, toluene, $\mathrm{CCl}_{4}$ gave lower yields under similar reaction conditions (Table 1, entries 1-3). The polar aprotic solvents $\left(\mathrm{CH}_{2} \mathrm{Cl}_{2}\right.$, THF and $\left.\mathrm{CH}_{3} \mathrm{CN}\right)$ also afforded comparatively lower yields (Table 1, entries 4-6). The polar protic solvents $\left(\mathrm{C}_{2} \mathrm{H}_{5} \mathrm{OH}, \mathrm{CH}_{3} \mathrm{OH}\right.$, PEG-400 and $\mathrm{H}_{2} \mathrm{O}$ ) still gave comparatively lower conversions (Table 1 , entries 7-10). It is remarkable that the reaction carried out at $60^{\circ} \mathrm{C}$ in the absence of catalyst and solvent afforded 3a in excellent yield (91\%), which is significantly higher than those obtained for the nonpolar or polar solvents. The structure of 3a was characterized by ${ }^{1} \mathrm{H}$ NMR, ${ }^{13} \mathrm{C}$ NMR, IR and by comparison with authentic samples prepared by literature procedure. The ${ }^{1} \mathrm{H}$ NMR spectra of $\mathbf{3 a}$ shows a characteristic peak at $\delta 7.87 \mathrm{ppm}$ corresponding to the hydrogen of imidazole ring, whereas in the ${ }^{13} \mathrm{C}$ NMR spectrum, the peak appearing of $\delta 108.1,145.7$ and 145.8 ppm corresponds to C-3, C-2 and C-9, respectively, of the imidazole ring. And in the IR spectrum, the structure of 3a showed $\mathrm{C}=\mathrm{N}$ stretching peak at $1625 \mathrm{~cm}^{-1}$.

With the optimized conditions in hand, the reactions of different 2-aminopyridines with various $\alpha$-haloketones were examined to explore the scope and generality of this present protocol for the synthesis of various imidazo[1,2-a] pyridines (Table 2). As expected, the reaction proceeded smoothly with yields ranging from good to excellent and tolerated various functional groups such as chloro, methyl and methoxy groups.

As shown in Table 2, the $\alpha$-bromoacetophenone with electron-rich functionality as well as electron-poor functionality undergoes condensation reaction with 2-aminopyridine or substituted 2-aminopyridine equally well to afford the corresponding products in good to excellent yields. Even when employing the hindered $\alpha$-methylphenacyl bromide and 2-aminopyridine or 2-amino-5-methylpyridine, good yields (Table 2, entries $10-11,70 \%$ and $64 \%$, respectively) were also obtained after slightly prolonging the reaction time to $60 \mathrm{~min}$. Nevertheless, 2-aminopyridine with electron-withdrawing substituted group chloro is less nucleophilic and more slowly than electron-neutral or donating analogues.

Encouraged by our success, we screened a few reactions of the commercially available and deactivated $\alpha$-chloroketone with 2 -aminopyridine or 2 -amino5 -methylpyridine. To our delight, when employing $\alpha$-chloroacetophenone and 2-aminopyridine or 2-amino5-methylpyridine, excellent yields (Table 2, entries 12-13, $83 \%$ and $88 \%$, respectively) were obtained after slightly prolonging the reaction time to $80 \mathrm{~min}$ and $40 \mathrm{~min}$, respectively. Furthermore, the para- and ortho-substituted 2,2',4'-trichloroacetophenone with 2-aminopyridine or 2-amino-5-methylpyridine also can afford the corresponding products $\mathbf{3 l}$ and $\mathbf{3 m}$ in $75 \%$ and $65 \%$ yields, respectively (Table 2, entries 14-15). 
Table 2. Synthesis of imidazo[1,2-a]pyridinepyrazoles under catalyst- and solvent-free conditions ${ }^{\mathrm{a}}$

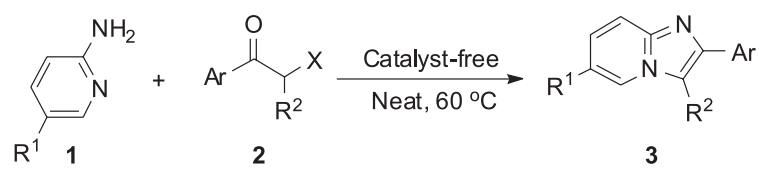

\begin{tabular}{|c|c|c|c|c|c|c|c|c|}
\hline \multirow{2}{*}{ Entry } & \multicolumn{3}{|c|}{$\alpha$-Haloketone } & \multirow{2}{*}{$\mathrm{R}^{1}$} & \multirow{2}{*}{ time $/ \mathrm{min}$} & \multirow{2}{*}{ Product } & \multirow{2}{*}{ Yield / $(\%)^{\mathrm{b}}$} & \multirow{2}{*}{$\mathrm{mp} /{ }^{\circ} \mathrm{C}$} \\
\hline & $\mathrm{Ar}$ & $X$ & $\mathrm{R}^{2}$ & & & & & \\
\hline 1 & $\mathrm{C}_{6} \mathrm{H}_{5}$ & $\mathrm{Br}$ & $\mathrm{H}$ & $\mathrm{H}$ & 20 & $\mathbf{3 a}$ & 91 & $136-137$ \\
\hline 2 & $\mathrm{C}_{6} \mathrm{H}_{5}$ & $\mathrm{Br}$ & $\mathrm{H}$ & $\mathrm{CH}_{3}$ & 5 & $3 \mathbf{b}$ & 92 & $171-173$ \\
\hline 3 & $\mathrm{C}_{6} \mathrm{H}_{5}$ & $\mathrm{Br}$ & $\mathrm{H}$ & $\mathrm{Cl}$ & 15 & $3 c$ & 90 & 204-206 \\
\hline 4 & $p-\mathrm{MeOC}_{6} \mathrm{H}_{4}$ & $\mathrm{Br}$ & $\mathrm{H}$ & $\mathrm{H}$ & 10 & 3d & 95 & $135-136$ \\
\hline 5 & $p-\mathrm{MeOC}_{6} \mathrm{H}_{4}$ & $\mathrm{Br}$ & $\mathrm{H}$ & $\mathrm{CH}_{3}$ & 5 & $\mathbf{3 e}$ & 93 & $179-181$ \\
\hline 6 & $p-\mathrm{MeOC}_{6} \mathrm{H}_{4}$ & $\mathrm{Br}$ & $\mathrm{H}$ & $\mathrm{Cl}$ & 25 & 3f & 80 & $234-236$ \\
\hline 7 & $p-\mathrm{ClC}_{6} \mathrm{H}_{4}$ & $\mathrm{Br}$ & $\mathrm{H}$ & $\mathrm{H}$ & 15 & $3 g$ & 95 & 207-209 \\
\hline 8 & $p-\mathrm{ClC}_{6} \mathrm{H}_{4}$ & $\mathrm{Br}$ & $\mathrm{H}$ & $\mathrm{CH}_{3}$ & 5 & $3 \mathbf{h}$ & 91 & $239-240$ \\
\hline 9 & $p-\mathrm{ClC}_{6} \mathrm{H}_{4}$ & $\mathrm{Br}$ & $\mathrm{H}$ & $\mathrm{Cl}$ & 130 & $\mathbf{3 i}$ & 75 & $205-207$ \\
\hline 10 & $\mathrm{C}_{6} \mathrm{H}_{5}$ & $\mathrm{Br}$ & $\mathrm{CH}_{3}$ & $\mathrm{H}$ & 60 & $\mathbf{3 j}$ & 70 & $159-161$ \\
\hline 11 & $\mathrm{C}_{6} \mathrm{H}_{5}$ & $\mathrm{Br}$ & $\mathrm{CH}_{3}$ & $\mathrm{CH}_{3}$ & 60 & $3 \mathbf{k}$ & 64 & $114-116$ \\
\hline 12 & $\mathrm{C}_{6} \mathrm{H}_{5}$ & $\mathrm{Cl}$ & $\mathrm{H}$ & $\mathrm{H}$ & 80 & $3 \mathbf{a}$ & 83 & $136-137$ \\
\hline 13 & $\mathrm{C}_{6} \mathrm{H}_{5}$ & $\mathrm{Cl}$ & $\mathrm{H}$ & $\mathrm{CH}_{3}$ & 40 & $3 \mathbf{b}$ & 88 & $171-173$ \\
\hline 14 & $2,4-\mathrm{Cl}_{2} \mathrm{C}_{6} \mathrm{H}_{3}$ & $\mathrm{Cl}$ & $\mathrm{H}$ & $\mathrm{H}$ & 50 & 31 & 75 & $181-182$ \\
\hline 15 & $2,4-\mathrm{Cl}_{2} \mathrm{C}_{6} \mathrm{H}_{3}$ & $\mathrm{Cl}$ & $\mathrm{H}$ & $\mathrm{CH}_{3}$ & 50 & $3 \mathrm{~m}$ & 65 & $135-136$ \\
\hline
\end{tabular}

a Reaction conditions: $\alpha$-haloketones $(1.0 \mathrm{mmol}), 2$-aminopyridines $(1.0 \mathrm{mmol})$ at $60{ }^{\circ} \mathrm{C}$. ${ }^{\mathrm{b}}$ Isolated yield.

In summary, we have described a simple, highly efficient, and facile procedure for the synthesis of imidazo[1,2- $a]$ pyridine derivatives from the readily available starting materials. To the best of our knowledge, this is the first report catalyst-free synthesis of imidazo[1,2-a]pyridines in the absence of solvent under mild conditions. The procedure offers simple experimental procedure, short reaction time, catalyst-free, solvent free, low cost, efficient yield and mild reaction conditions, which makes this method a useful and attractive strategy in view of economic and environmental advantages. Currently, studies on the extension of this protocol are ongoing in our laboratory.

\section{Experimental}

All reagents were commercial available and used without any purification. Melting points were recorded on Digital Melting Point Apparatus WRS-1B and are uncorrected. IR spectra were recorded on a Bruker-EQUINOX55 spectrometer. Mass spectra (EI, $70 \mathrm{ev}$ ) were measured with SHIMADZU GCMS-QP2010 Plus. ${ }^{1} \mathrm{H}$ NMR and ${ }^{13} \mathrm{C}$ NMR spectra were recorded on a Brucker AC 300 instrument using $\mathrm{CDCl}_{3}$ as the solvent with tetramethylsilane (TMS) as an internal standard at room temperature. Chemical shifts were given in $\delta$ relative to TMS, the coupling constants $J$ are given in Hz. Elemental analysis was determined on a CarloErba 1108 instrument. All reactions were conducted using standard Schlenk techniques. Column chromatography was performed using EM Silica gel 60 (300-400 mesh).

\section{General procedure for the preparation of pyrazoles}

A mixture of $\alpha$-haloketone 2 $(1.0 \mathrm{mmol}), 2$-aminopyridine or substituted 2-aminopyridine $\mathbf{1}(1.0 \mathrm{mmol})$ was stirred at $60{ }^{\circ} \mathrm{C}$ under vigorous magnetic stirring for the specified time as mentioned in Table 2. The progress of the reaction was monitored by TLC. After completion of the reaction, the product was washed with dehydrated alcohol or ethyl acetate $(3 \times 10 \mathrm{~mL})$. The combined organic solvent was removed under vacuum to obtain the crude solid product. The crude product was further purified by silica gel column chromatography using ethyl acetate-petroleum ether (1:3) as eluent to afford the pure product 3 .

The spectral and analytical data of all compounds are given below.

\section{2-Phenylimidazo[1,2-a]pyridine (3a)}

White solid, mp 136- $137{ }^{\circ} \mathrm{C}$ (lit. 131-133 ${ }^{\circ} \mathrm{C}$ ) $;{ }^{20}{ }^{1} \mathrm{H}$ NMR $\left(300 \mathrm{MHz}, \mathrm{CDCl}_{3}\right) \delta(\mathrm{ppm}) 8.12(\mathrm{dd}, J 1.04,1.03,1 \mathrm{H})$, 7.98-7.96 (m, 2H), $7.88(\mathrm{~s}, 1 \mathrm{H}), 7.64(\mathrm{~d}, J 9.14,1 \mathrm{H}), 7.45$ 
(t, J 7.22, 2H), $7.35(\mathrm{~d}, J 7.33,1 \mathrm{H}), 7.20-7.17(\mathrm{~m}, 1 \mathrm{H}), 6.79$ $(\mathrm{d}, J 6.78,1 \mathrm{H}) ;{ }^{13} \mathrm{C}$ NMR $\left(75 \mathrm{MHz}, \mathrm{CDCl}_{3}\right) \delta$ (ppm) 145.8, 145.7, 133.8, 128.7, 127.9, 126.0, 125.6, 124.6, 117.5, 112.4, 108.1. IR (KBr) $v_{\max } / \mathrm{cm}^{-1}: 2925,2857,1738,1625$, 1511, 1460, 1383, 1269, 1201, 1122, 1081, 1040, 744, 688, 458. MS (ESI): $m / z(\%) 195\left([\mathrm{M}+\mathrm{H}]^{+}, 100\right)$.

\section{6-Methyl-2-phenylimidazo[1,2-a]pyridine (3b)}

White solid, mp 171-173 ${ }^{\circ} \mathrm{C}$ (lit. 172-174 ${ }^{\circ} \mathrm{C}$ ) ${ }^{24}{ }^{1} \mathrm{H}$ NMR $\left(300 \mathrm{MHz}, \mathrm{CDCl}_{3}\right) \delta(\mathrm{ppm}) 7.95(\mathrm{~d}, J 7.05,2 \mathrm{H}), 7.83$ (s, $1 \mathrm{H}), 7.73(\mathrm{~s}, 1 \mathrm{H}), 7.53(\mathrm{~d}, J 9.09,1 \mathrm{H}), 7.44(\mathrm{t}, J 6.63$, $2 \mathrm{H}), 7.33(\mathrm{~d}, J 6.44,1 \mathrm{H}), 7.00(\mathrm{~d}, J 8.82,1 \mathrm{H}), 2.29(\mathrm{~s}, 3 \mathrm{H}$, $\left.\mathrm{CH}_{3}\right) ;{ }^{13} \mathrm{C}$ NMR (75 MHz, $\left.\mathrm{CDCl}_{3}\right) \delta$ (ppm) 145.4, 144.7, 133.9, 128.7, 127.84, 127.78, 125.9, 123.3, 122.0, 116.7, 107.9, 18.0; IR (KBr) $v_{\max } / \mathrm{cm}^{-1}: 2921,1628,1525,1471$, 1418, 1342, 1259, 1206, 1159, 1079, 846, 805, 768, 715, 685, 571, 506 .

\section{6-Chloro-2-phenylimidazo[1,2-a]pyridine (3c)}

White solid, mp 204-206 ${ }^{\circ} \mathrm{C}$ (lit. 204-207 ${ }^{\circ} \mathrm{C}$ ) $;{ }^{25}{ }^{1} \mathrm{H}$ NMR (300 MHz, $\left.\mathrm{CDCl}_{3}\right) \delta(\mathrm{ppm}) 8.15(\mathrm{~s}, 1 \mathrm{H}), 7.94$ (d, $J$ 7.25, 2H), $7.82(\mathrm{~s}, 1 \mathrm{H}), 7.57(\mathrm{~d}, J 9.12,1 \mathrm{H}), 7.51-7.38(\mathrm{~m}$, $2 \mathrm{H}), 7.34(\mathrm{~d}, J 7.19,1 \mathrm{H}), 7.14(\mathrm{~d}, J 9.41,1 \mathrm{H}) ;{ }^{13} \mathrm{C} \mathrm{NMR}$ $\left(75 \mathrm{MHz}, \mathrm{CDCl}_{3}\right) \delta(\mathrm{ppm}) 146.9,144.1,133.3,128.8$, 128.3, 126.1, 126.0, 123.4, 120.5, 117.9, 108.5; IR (KBr) $\mathrm{V}_{\max } / \mathrm{cm}^{-1}: 2976,2925,1635,1512,1473,1425,1387,1332$, 1241, 1206, 1133, 1074, 938, 809, 772, 719, 506.

\section{2-(4-Methoxyphenyl)imidazo[1,2-a]pyridine (3d)}

White solid, mp $135-136{ }^{\circ} \mathrm{C}$ (lit. 133-134 $\left.{ }^{\circ} \mathrm{C}\right) ;{ }^{17} \mathrm{H}$ NMR $\left(300 \mathrm{MHz}, \mathrm{CDCl}_{3}\right) \delta(\mathrm{ppm}) 8.09(\mathrm{~d}, J 6.77,1 \mathrm{H}), 7.89(\mathrm{dd}$, $J 1.95,1.94,2 \mathrm{H}), 7.77$ (s, 1H), 7.61 (d, $J 9.08,1 \mathrm{H}), 7.17$ 7.15 (m, 1H), 6.97 (dd, J 1.97, 1.94, 2H), 6.76 (dd, J 0.82, $0.81,1 \mathrm{H}), 3.85\left(\mathrm{~s}, 3 \mathrm{H}, \mathrm{OCH}_{3}\right) ;{ }^{13} \mathrm{C} \mathrm{NMR}\left(75 \mathrm{MHz}, \mathrm{CDCl}_{3}\right)$ $\delta$ (ppm) 159.6, 145.64, 145.57, 127.3, 126.4, 125.4, 124.5, 117.2, 114.1, 112.3, 107.2, 55.3; IR (KBr) $v_{\max } / \mathrm{cm}^{-1}: 2961$, 2838, 1612, 1548, 1482, 1371, 1285, 1244, 1175, 1110 , 1077, 1030, 924, 838, 743, 631, 536, 446.

2-(4-Methoxyphenyl)-6-methylimidazo[1,2-a]pyridine (3e)

White solid, mp 179-181 ${ }^{\circ} \mathrm{C}$; ${ }^{1} \mathrm{H}$ NMR $(300 \mathrm{MHz}$, $\left.\mathrm{CDCl}_{3}\right) \delta(\mathrm{ppm}) 7.88(\mathrm{~d}, J 2.26,2 \mathrm{H}), 7.86(\mathrm{~d}, J 2.11,1 \mathrm{H})$, $7.68(\mathrm{~s}, 1 \mathrm{H}), 7.51(\mathrm{~d}, J 9.48,1 \mathrm{H}), 7.01(\mathrm{~d}, J 1.57,2 \mathrm{H}), 6.96$ (dd, $J$ 2.10, 2.06, 1H), 3.85 (s, 3H, $\left.\mathrm{OCH}_{3}\right), 2.31$ (s, 3H, $\left.\mathrm{CH}_{3}\right) ;{ }^{13} \mathrm{C}$ NMR (75 MHz, $\mathrm{CDCl}_{3}$ ) $\delta$ (ppm) 159.5, 145.3, 144.6, 127.7, 127.2, 126.6, 123.3, 121.9, 116.5, 114.1, 107.0, 55.3, 18.1; IR (KBr) $v_{\max } / \mathrm{cm}^{-1}: 2926,1612,1546$, 1483, 1413, 1341, 1301, 1246, 1174, 1103, 1023, 839, 794, 744, 710, 591, 528; MS (ESI): $m / z(\%), 239\left([\mathrm{M}+\mathrm{H}]^{+}, 100\right)$; Anal. calc. for $\mathrm{C}_{15} \mathrm{H}_{14} \mathrm{~N}_{2} \mathrm{O}$ : C, 75.61; H, 5.92; N, 11.76. Found: C, 75.63; H, 5.90; N, 11.78 .
6-Chloro-2-(4-methoxyphenyl)imidazo[1,2-a]pyridine (3f)

White solid, mp $234-236{ }^{\circ} \mathrm{C}$ (lit. 227-228 ${ }^{\circ} \mathrm{C}$ ) ${ }^{26}{ }^{1} \mathrm{H}$ NMR (300 MHz, $\left.\mathrm{CDCl}_{3}\right) \delta(\mathrm{ppm}) 8.16-8.15(\mathrm{~m}, 1 \mathrm{H}), 7.88$ (dd, $J$ $2.05,2.04,2 \mathrm{H}), 7.75(\mathrm{~s}, 1 \mathrm{H}), 7.56(\mathrm{~d}, J 9.54,1 \mathrm{H}), 7.13(\mathrm{dd}$, $J 1.99,1.96,1 \mathrm{H}$ ), 6.99 (dd, $J 2.00,2.01,2 \mathrm{H}), 3.87$ (s, 3H, $\left.\mathrm{OCH}_{3}\right) ;{ }^{13} \mathrm{C}$ NMR $\left(75 \mathrm{MHz}, \mathrm{CDCl}_{3}\right) \delta$ (ppm) 160.1, 147.1, 144.2, 127.6, 126.3, 126.0, 123.4, 120.5, 117.8, 114.4, 107.8, 55.5. IR (KBr) $v_{\text {max }} / \mathrm{cm}^{-1}: 2995,1610,1551,1487,1371$, 1305, 1250, 1176, 1108, 1070, 1030, 933, 841, 803, 742, 705, 575, 524; MS (ESI): $m / z(\%), 259\left([\mathrm{M}+\mathrm{H}]^{+}, 100\right)$.

\section{2-(4-Chlorophenyl)imidazo[1,2-a]pyridine (3g)}

White solid, mp 207-209 ${ }^{\circ} \mathrm{C}$ (lit. $201^{\circ} \mathrm{C}$ ) ${ }^{18}{ }^{1} \mathrm{H}$ NMR (300 $\left.\mathrm{MHz}, \mathrm{CDCl}_{3}\right) \delta(\mathrm{ppm}) 8.09(\mathrm{~d}, J 6.78,1 \mathrm{H}), 7.87(\mathrm{~d}, J 8.52,2 \mathrm{H})$, $7.82(\mathrm{~s}, 1 \mathrm{H}), 7.61(\mathrm{~d}, J 9.15,1 \mathrm{H}), 7.39(\mathrm{~d}, J 8.52,2 \mathrm{H}), 7.17(\mathrm{t}$, $J 7.17,1 \mathrm{H}), 6.77(\mathrm{t}, J 6.60,1 \mathrm{H}) ;{ }^{13} \mathrm{C} \mathrm{NMR}\left(75 \mathrm{MHz}, \mathrm{CDCl}_{3}\right)$ $\delta(\mathrm{ppm})$ 145.7, 144.6, 133.6, 132.3, 128.9, 127.2, 125.6, 124.9, 117.5, 112.6, 108.2; IR (KBr) $v_{\text {max }} / \mathrm{cm}^{-1}: 2917,1632,1471$, 1369, 1250, 1202, 1089, 1009, 933, 830, 742, 598, 510.

2-(4-Chlorophenyl)-6-methylimidazo[1,2-a]pyridine (3h)

White solid, mp 239-240 ${ }^{\circ} \mathrm{C}$ (lit. 240-242 $\left.{ }^{\circ} \mathrm{C}\right) ;{ }^{15} \mathrm{H}$ NMR $\left(300 \mathrm{MHz}, \mathrm{CDCl}_{3}\right) \delta(\mathrm{ppm}) 7.89(\mathrm{~d}, J 2.83,2 \mathrm{H}), 7.88(\mathrm{~s}$, $1 \mathrm{H}), 7.75(\mathrm{~s}, 1 \mathrm{H}), 7.54(\mathrm{t}, J 6.05,1 \mathrm{H}), 7.39(\mathrm{~d}, J 8.53,2 \mathrm{H})$, $7.05(\mathrm{~d}, J 9.34,1 \mathrm{H}), 2.33\left(\mathrm{~s}, 3 \mathrm{H}, \mathrm{CH}_{3}\right){ }^{13} \mathrm{C} \mathrm{NMR}(75 \mathrm{MHz}$, $\left.\mathrm{CDCl}_{3}\right) \delta(\mathrm{ppm}) 144.8,144.4,133.5,132.5,128.9,128.1$, 127.1, 123.3, 122.3, 116.8, 107.9, 18.1; IR (KBr) $v_{\text {max }} / \mathrm{cm}^{-1}$ : 2921, 1635, 1540, 1465, 1409, 1257, 1206, 1090, 1010, 944, 835, 803, 734, 511.

6-Chloro-2-(4-chlorophenyl)imidazo[1,2-a]pyridine (3i)

White solid, mp 205-207 ${ }^{\circ} \mathrm{C}$ (lit. $209{ }^{\circ} \mathrm{C}$ ) $;{ }^{27}{ }^{1} \mathrm{H}$ NMR (300 MHz, $\left.\mathrm{CDCl}_{3}\right) \delta(\mathrm{ppm}) 8.14-8.13(\mathrm{~m}, 1 \mathrm{H}), 7.85$ (dd, $J$ $1.99,1.97,2 \mathrm{H}), 7.77(\mathrm{~s}, 1 \mathrm{H}), 7.55(\mathrm{~d}, J 9.57,1 \mathrm{H}), 7.42-7.39$ $(\mathrm{m}, 2 \mathrm{H}), 7.14$ (dd, $J 1.98,1.97,1 \mathrm{H}) ;{ }^{13} \mathrm{C}$ NMR $(75 \mathrm{MHz}$, $\left.\mathrm{CDCl}_{3}\right) \delta$ (ppm) 145.9, 144.3, 134.3, 132.0, 129.2, 127.5, 126.6, 123.6, 121.0, 118.1, 108.7; IR (KBr) $v_{\text {max }} / \mathrm{cm}^{-1}: 3130$, 1653, 1525, 1470, 1419, 1336, 1260, 1202, 1071, 1009, 935, 833, 799, 734, 511; MS (ESI): $m / z(\%), 263\left([\mathrm{M}+\mathrm{H}]^{+}\right.$, 100), $265\left([\mathrm{M}+2+\mathrm{H}]^{+}, 70\right), 267\left([\mathrm{M}+4+\mathrm{H}]^{+}, 15\right)$.

\section{3-Methyl-2-phenylimidazo[1,2-a]pyridine (3j)}

Pale yellow solid, mp $159-161{ }^{\circ} \mathrm{C}$ (lit. $\left.153-154{ }^{\circ} \mathrm{C}\right) ;{ }^{17}$ ${ }^{1} \mathrm{H}$ NMR $\left(300 \mathrm{MHz}, \mathrm{CDCl}_{3}\right) \delta(\mathrm{ppm}) 7.88(\mathrm{~d}, J 6.87,1 \mathrm{H})$, 7.82-7.78 (m, 2H), $7.64(\mathrm{~d}, J 9.06,1 \mathrm{H}), 7.49-7.44(\mathrm{~m}, 2 \mathrm{H})$, $7.36(\mathrm{~d}, J 7.38,1 \mathrm{H}), 7.17-7.16(\mathrm{~m}, 1 \mathrm{H}), 6.84(\mathrm{~d}, J 6.75$, $1 \mathrm{H}), 2.63\left(\mathrm{~s}, 3 \mathrm{H}, \mathrm{CH}_{3}\right) ;{ }^{13} \mathrm{CNMR}\left(75 \mathrm{MHz}, \mathrm{CDCl}_{3}\right) \delta(\mathrm{ppm})$ $144.3,142.4,134.9,128.5,128.3,127.3,123.5,122.8$, 117.4, 115.9, 112.0, 9.6; IR (KBr) v $v_{\max } / \mathrm{cm}^{-1}: 3027,1629$, 1494, 1443, 1393, 1351, 1243, 1143, 1072, 909, 751, 697, 583, 505; MS (ESI): $m / z(\%), 209\left([\mathrm{M}+\mathrm{H}]^{+}, 100\right)$. 
3,6-Dimethyl-2-phenylimidazo[1,2-a]pyridine (3k)

Pale yellow solid, mp $114-116^{\circ} \mathrm{C} ;{ }^{1} \mathrm{H}$ NMR $(300 \mathrm{MHz}$, $\left.\mathrm{CDCl}_{3}\right) \delta(\mathrm{ppm}) 7.82-7.79(\mathrm{~m}, 2 \mathrm{H}), 7.66(\mathrm{~s}, 1 \mathrm{H}), 7.54(\mathrm{~d}$, $J$ 9.16, 1H), $7.46(\mathrm{t}, J 7.36,2 \mathrm{H}), 7.34$ (t, $J 7.49,1 \mathrm{H}), 7.02$ $(\mathrm{d}, J 9.17,1 \mathrm{H}), 2.61\left(\mathrm{~s}, 3 \mathrm{H}, \mathrm{CH}_{3}\right), 2.36\left(\mathrm{~s}, 3 \mathrm{H}, \mathrm{CH}_{3}\right) ;{ }^{13} \mathrm{C}$ NMR (75 MHz, $\left.\mathrm{CDCl}_{3}\right) \delta(\mathrm{ppm}) 143.5,142.3,135.1,128.5$, 128.2, 127.2, 126.6, 121.5, 120.6, 116.8, 115.6, 18.4, 9.7; IR (KBr) $v_{\text {max }} / \mathrm{cm}^{-1}: 2920,1634,1496,1447,1388,1332$, 1261, 1188, 1127, 1043, 777, 699, 581, 507; MS (ESI): $\mathrm{m} / 2$ (\%), $223\left([\mathrm{M}+\mathrm{H}]^{+}, 100\right)$; Anal. calc. for $\mathrm{C}_{15} \mathrm{H}_{14} \mathrm{~N}_{2}: \mathrm{C}, 81.05$; H, 6.35; N, 12.60. Found: C, 81.08; H, 6.34; N, 12.58 .

\section{2-(2,4-Dichlorophenyl)imidazo[1,2-a]pyridine (3l)}

Pale yellow solid, mp $181-182{ }^{\circ} \mathrm{C} ;{ }^{1} \mathrm{H}$ NMR $(300 \mathrm{MHz}$, $\left.\mathrm{CDCl}_{3}\right) \delta(\mathrm{ppm})$ 8.28-8.26 (m, 2H), $8.14(\mathrm{~d}, J 6.77,1 \mathrm{H})$, $7.62(\mathrm{~d}, J 9.11,1 \mathrm{H}), 7.49-7.48(\mathrm{~m}, 1 \mathrm{H}), 7.36(\mathrm{dd}, J 2.01$, 1.99, 1H), 7.23-7.18 (m, 1H), $6.81(\mathrm{t}, J 6.76,1 \mathrm{H}) ;{ }^{13} \mathrm{C} \mathrm{NMR}$ $\left(75 \mathrm{MHz}, \mathrm{CDCl}_{3}\right) \delta(\mathrm{ppm}) 144.5,140.8,133.6,132.1$, $131.7,130.9,130.0,127.4,125.8,125.1,117.6,112.6$, 112.4; IR (KBr) $v_{\max } / \mathrm{cm}^{-1}: 3042,1632,1544,1461,1367$, 1242, 1105, 1043, 931, 826, 749, 704, 623, 555; MS (ESI): $\mathrm{m} / \mathrm{z}(\%), 263\left([\mathrm{M}+\mathrm{H}]^{+}, 100\right), 265\left([\mathrm{M}+2+\mathrm{H}]^{+}, 70\right), 267$ $\left([\mathrm{M}+4+\mathrm{H}]^{+}, 15\right)$; Anal. calc. for $\mathrm{C}_{15} \mathrm{H}_{12} \mathrm{Cl}_{2} \mathrm{~N}_{2}: \mathrm{C}, 61.87 ; \mathrm{H}$, 4.15; N, 9.62. Found: C, 61.88; H, 4.17; N, 9.59.

\section{2-(2,4-Dichlorophenyl)-6-methylimidazo[1,2-a]pyridine} (3m)

Pale yellow solid, mp $134-135{ }^{\circ} \mathrm{C} ;{ }^{1} \mathrm{H}$ NMR $(300 \mathrm{MHz}$, $\left.\mathrm{CDCl}_{3}\right) \delta(\mathrm{ppm}) 8.25(\mathrm{~d}, J 8.55,1 \mathrm{H}), 8.17(\mathrm{~s}, 1 \mathrm{H}), 7.91(\mathrm{~s}$, $1 \mathrm{H}), 7.51(\mathrm{~d}, J 9.33,1 \mathrm{H}), 7.46(\mathrm{~d}, J 2.13,1 \mathrm{H}), 7.34(\mathrm{dd}$, $J$ 2.16, 2.07, 1H), $7.04(\mathrm{dd}, J 1.47,1.50,1 \mathrm{H}) ;{ }^{13} \mathrm{C} \mathrm{NMR}$ $\left(75 \mathrm{MHz}, \mathrm{CDCl}_{3}\right) \delta$ (ppm) 143.6, 140.5, 133.4, 132.0, 131.6, 131.1, 130.0, 128.3, 127.3, 123.4, 122.2, 116.8, 112.2, 18.1; IR (KBr) $v_{\max } / \mathrm{cm}^{-1}: 2960,1641,1540,1458$, 1419, 1380, 1342, 1256, 1201, 1142, 1096, 1039, 938, 853, 793, 743, 700, 563, 461; MS (ESI): $m / z(\%), 277\left([\mathrm{M}+\mathrm{H}]^{+}\right.$, 100), $279\left([\mathrm{M}+2+\mathrm{H}]^{+}, 70\right), 281\left([\mathrm{M}+4+\mathrm{H}]^{+}, 15\right)$; Anal. calc. for $\mathrm{C}_{14} \mathrm{H}_{10} \mathrm{Cl}_{2} \mathrm{~N}_{2}: \mathrm{C}, 60.67 ; \mathrm{H}, 3.64 ; \mathrm{N}, 10.11$. Found: C, $60.69 ; \mathrm{H}, 3.65 ; \mathrm{N}, 10.09$.

\section{Acknowledgments}

We are grateful to the National Key Technology R\&D Program (No. 2007BAI34B00) and the Natural Science Foundation of Zhengjiang Province (No. Y4080107) for financial support.

\section{Supplementary Information}

Supplementary data are available free of charge at http:// jbcs.sbq.org.br, as PDF file.

\section{References}

1. Gudmundsson, K. S.; Johns, B. A.; Org. Lett. 2003, 5, 1369; Enguehard, C.; Fauvelle, F.; Debouzy, J.; Peinnequin, A.; Thery, I.; Dabouis V.; Gueiffier, A.; Eur. J. Pharm. Sci. 2005, 24, 219; Hamdouchi, C.; Zhong, B.; Mendoza, J.; Collins, E.; Jaramillo, C.; Diego, J.; Robertson, D.; Spencer, C. D.; Anderson, B. D.; Watkins, S. A.; Zhang F.; Brooks, H. B.; Bioorg. Med. Chem. Lett. 2005, 15, 1943.

2. Elhakmoui, A.; Gueiffier, A.; Milhavet, J. C.; Blache Y.; Chapat, J. P.; Bioorg. Med. Chem. Lett. 1994, 4, 1937; Gueiffier, A.; Lhassani, M.; Elhakmoui, A.; Snoeck, R.; Andrei, G.; Chavignion, O.; Teulade, J. C.; Kerbal, A.; Essassi, M.; Debouzy, J. C.; Witurowo, J. P.; Blache, Y.; Balzarini, J.; De Clercq, E.; Chapat, J. P.; J. Med. Chem. 1996, 39, 2856; Gudmundsson, K. S.; Drach J. C.; Townsend, L. B.; J. Org. Chem. 1997, 62, 3453; Pan, S.; Wang, G.; Shinazi, R. F.; Zhao, K.; Tetrahedron Lett. 1998, 39, 8191; Hamdouchi, C.; de Blas, J.; del Prado, M.; Gruber, J.; Heinz, B. A.; Vance, L.; J. Med. Chem. 1999, 42, 50; Gudmundsson, K. S.; Williams, J. D.; Drach, J. C.; Townsend, L.; B. J. Med. Chem. 2003, 46, 1449; Puerstinger, G.; Paeshuyse, J.; Declercq, E.; Neyts, J.; Bioorg. Med. Chem. Lett. 2007, 17, 390.

3. Kaminsky, J. J.; Puchalski, C.; Solomon, D. M.; Rizvi, R. K.; Conn, D. J.; Elliot, A. J.; Lovey, R. G.; Guzik, H.; Chui, P. J. S.; Long, J. F.; McPhail, A. T.; J. Med. Chem. 1989, 32, 1686; Kaesura, Y.; Nishino, S.; Inoue, Y.; Tomoi, M.; Taksugi, H.; Chem. Pharm. Bull. 1992, 40, 371; Kaminsky, J. J.; Doweyko, A. M.; J. Med. Chem. 1997, 40, 427.

4. Rewankar, G. R.; Matthews, J. R.; Robins, R. K.; J. Med. Chem. 1975, 18, 1253; Rival, Y.; Grassy G.; Michel, G.; Chem. Pharm. Bull. 1992, 40, 1170.

5. Fisher, M. H.; Lusi, A.; J. Med. Chem. 1972, 15, 982; Rival, Y.; Grassy, G.; Taudou, A.; Ecalle, R.; Eur. J. Med. Chem. 1991, $26,13$.

6. Biftu, T.; Feng, D.; Fisher, M.; Liang, G. B.; Qian, X.; Scribner, A.; Dennis, R.; Lee, S.; Liberator, P. A.; Brown, C.; Gurnett, A.; Leavitt, P. S.; Thompson, D.; Mathew, J.; Misura, A.; Samaras, S.; Tamas, T.; Sina, J. F.; McNulty, K. A.; McKnight, C. G.; Bioorg. Med. Chem. Lett. 2006, 16, 2479; Ismail, M. A.; Arafa, R. K.; Wenzler, T.; Brun,R.; Tanious, F. A.; Wilson, W. D. Boykin, D. W.; Bioorg. Med. Chem. 2008, 16, 683.

7. Gudmundsson, K. S.; Johns, B. A.; Bioorg. Med. Chem. Lett. 2007, 17, 2735.

8. Rupert, K. C.; Henry, J. R.; Dodd, J. H.; Wadsworth, S. A.; Cavender, D. E.; Olini, G. C.; Fahmy; B.; Siekierka, J. J.; Bioorg. Med. Chem. Lett. 2003, 13, 347.

9. Leopoldo, M.; Lacivita, E.; Passafiume, E.; Contino, M.; Colabufo, N. A.; Berardi, F.; Perrone, R.; J. Med. Chem. 2007, 50, 5043.

10. Wright, J.; Heffner, T.; Pugsley, T.; MacKenzie, R.; Wise, L.; Bioorg. Med. Chem. Lett. 1995, 5, 2547. 
11. Burkholder, C.; Dolbier, Jr. W. R.; Médebielle, M.; Ait-Mohand, S.; Tetrahedron Lett. 2001, 42, 3077; Patel, H. S.; Linn, J. A.; Drewry, D. H.; Hillesheim, D. A.; Zuercher, W. J.; Hoekstra, W. J.; Tetrahedron Lett. 2003, 44, 4077; Ismail, M. A.; Brun, R.; Wenzler, T.; Tanious, F. A.; Wilson, W. D.; Boykin, D. W.; J. Med. Chem. 2004, 47, 3658; DiMauro E. F.; Vitullo, J. R.; J. Org. Chem. 2006, 71, 3959; Sharma, S.; Saha, B.; Sawant, D.; Kundu, B.; J. Comb. Chem. 2007, 9, 783.

12. Gudmundsson, K. S.; Johns, B. A.; Org. Lett. 2003, 5, 1369; Koubachi, J.; Kazzouli, S. E.; Berteina-Raboin, S.; Mouaddib A.; Guillaumet, G.; J. Org. Chem. 2007, 72, 7650; Takizawa, S.; Nishida, J.; Tsuzuki, T.; Tokito, S.; Yamashita, Y.; Inorg. Chem. 2007, 46, 4308.

13. Swakula, E.; Paudler, W.; Tetrahedron 1982, 38, 49; Cai, L.; Chin, F. T.; Pike, V. W.; Toyama, H.; Liow, J. S.; Zoghbi, S. S.; Modell, K.; Briard, E.; Shetty, H. U.; Sinclair, K.; Donohue, S.; Tipre, D.; Kung, M. P.; Dagostin, C.; Widdowson, D. A.; Green, M.; Gao, W.; Herman, M. M.; Ichise, M.; Innis, R. B.; J. Med. Chem. 2004, 47, 2208.

14. Kazzouli, S. E.; Berteina-Raboin, S.; Mouaddib, A. Guillaumet, G.; Tetrahedron Lett. 2003, 44, 6265.

15. Ponnala, S.; Kiran Kumar, S. T. V. S.; Bhat, B. A.; Sahu, D. P.; Synth. Commun. 2005, 901.

16. Cai, L.; Brouwer, C.; Sinclair, K.; Cuevas, J.; Pike, V. W.; Synthesis 2006, 133.

17. Xie, Y. Y.; Chen, Z. C.; Zheng, Q. G.; Synthesis 2002, 1505.

18. Ueno, M.; Togo, H.; Synthesis 2004, 2673.

19. Liu, Z.; Chen, Z. C.; Zheng, Q. G.; Synth. Commun. 2004, 361.
20. Yadav, J. S.; Reddy, B. V. S.; Rao, Y. G.; Srinivas, M.; Narsaiah, A. V.; Tetrahedron Lett. 2007, 48, 7717.

21. Bakherad, M.; Nasr-Isfahani, H.; Keivanloo, A.; Doostmohammadi, N.; Tetrahedron Lett. 2008, 49, 3819.

22. Dupont, J.; Consorti, C. S.; Spencer. J.; J. Braz. Chem. Soc. 2000, 11, 337; Lenardao, E. J.; Freitag, R. A.; Dabdoub, M. J.; Batista, A. C. F.; Silveira, C. C.; Quim. Nova 2003, 26, 123; Perin, G.; Jacob, R. G.; Botteselle, G. V.; Kublik, E. L.; Lenardão, E. J.; Cella, R.; dos Santos, P. C. S.; J. Braz. Chem. Soc. 2005, 16, 857.

23. Chen, J. X.; Wu, H. Y.; Zheng, Z. G.; Jin, C.; Zhang, X. X.; Su, W. K.; Tetrahedron Lett. 2006, 47, 5383; Su, W. K.; Chen, J. X.; Wu, H. Y.; Jin, C.; J. Org. Chem. 2007, 72, 4524; Chen, J. X.; Wu, D. Z.; He, F.; Liu M. C.; Wu H. Y.; Ding, J. C.; Su, W. K.; Tetrahedron Lett. 2008, 49, 3814; Chen, J. X.; Wu, H. Y.; Jin, C.; Zhang, X. X.; Xie, Y. Y.; Su, W. K.; Green Chem. 2006, 8, 330; Chen, X. A.; Zhang, C. F.; Wu, H. Y.; Yu, X. C.; Su, W. K.; Ding, J. C.; Synthesis 2007, 20, 3233; Chen, J. X.; Su, W. K.; Wu, H. Y.; Liu, M. C.; Jin, C.; Green Chem. 2007, 9, 972; Chen, J. X.; Liu, M. C.; Yang, X. L.; Ding, J. C.; Wu, H. Y.; J. Braz. Chem. Soc. 2008, 19, 877; Xiong, W.; Chen, J. X.; Liu, M. C.; Ding, J. C.; Wu, H. Y.; Su, W. K.; J. Braz. Chem. Soc. 2009, 20, 367.

24. Mattu, F.; Marongiu, E.; Ann. Chim. 1964, 5, 496.

25. Tomoda, H.; Hirano, T.; Saito, S.; Mutai, T.; Araki, K.; Bull. Chem. Soc. Jpn. 1999, 72, 1327.

26. Katritzky, A. R.; Qui, G.; Long, Q. H.; He, H. Y.; Steel, P. J.; J. Org. Chem. 2000, 65, 9201.

27. Buu-Hoi, N. P.; Xuong, N. D.; Suu, V. T.; J. Chem. Soc.1958, 2815 . 


\section{Catalyst- and Solvent-free Synthesis of Imidazo[1,2-a]pyridines}

\section{Dong-Jian Zhu, ${ }^{a}$ Jiu-Xi Chen, ${ }^{*, a}$ Miao-Chang Liu, ${ }^{a}$ Jin-Chang Ding, ${ }^{a, b}$ and Hua-Yue Wu ${ }^{*, a}$}

${ }^{a}$ College of Chemistry and Materials Engineering, Wenzhou University, Wenzhou, 325027, China

${ }^{b}$ Wenzhou Vocational and Technical College, Wenzhou, 325035, P. R. China
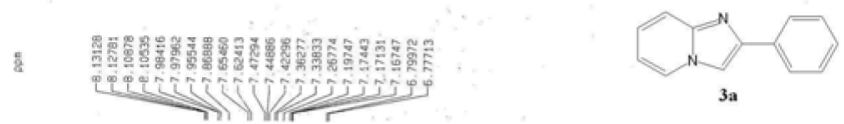

$3 \mathrm{a}$

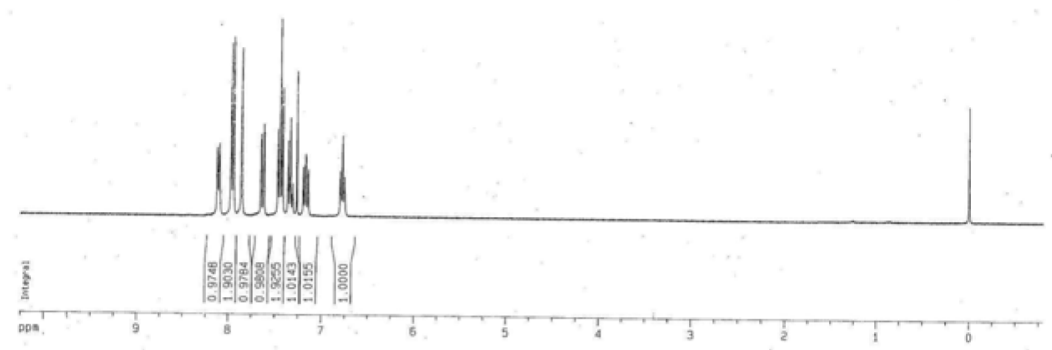

高
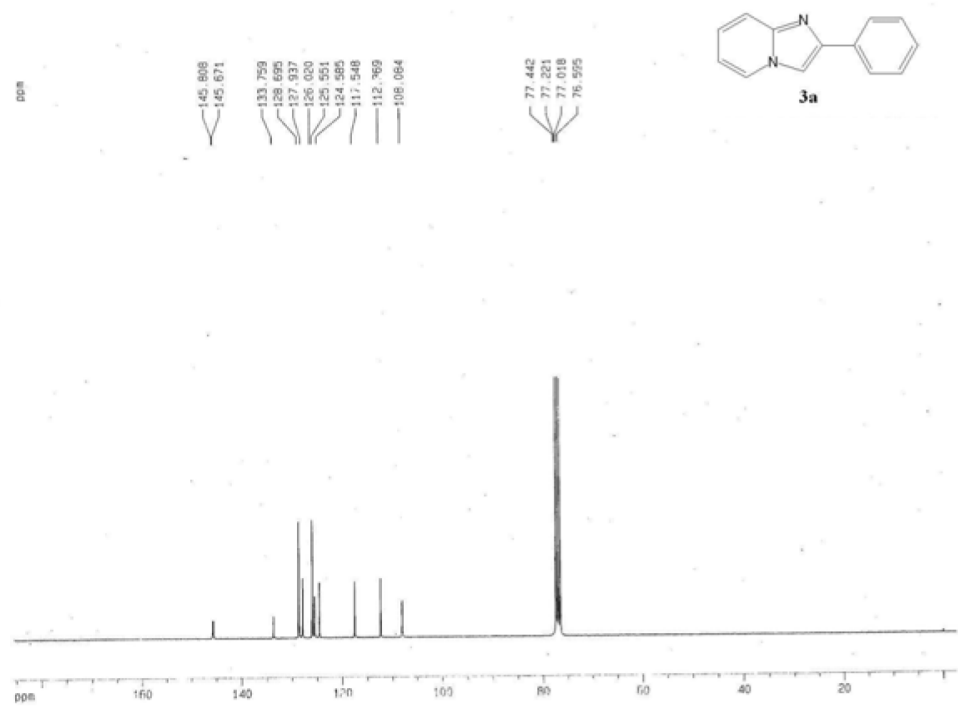

Figure S1. ${ }^{1} \mathrm{H}$ NMR of $\mathbf{3 a}\left(300 \mathrm{MHz}, \mathrm{CDCl}_{3}\right)$ and ${ }^{13} \mathrm{C} \mathrm{NMR}$ of $\mathbf{3 a}\left(75 \mathrm{MHz}, \mathrm{CDCl}_{3}\right)$.

*e-mail: jiuxichen@wzu.edu.cn; huayuewu@wzu.edu.cn 


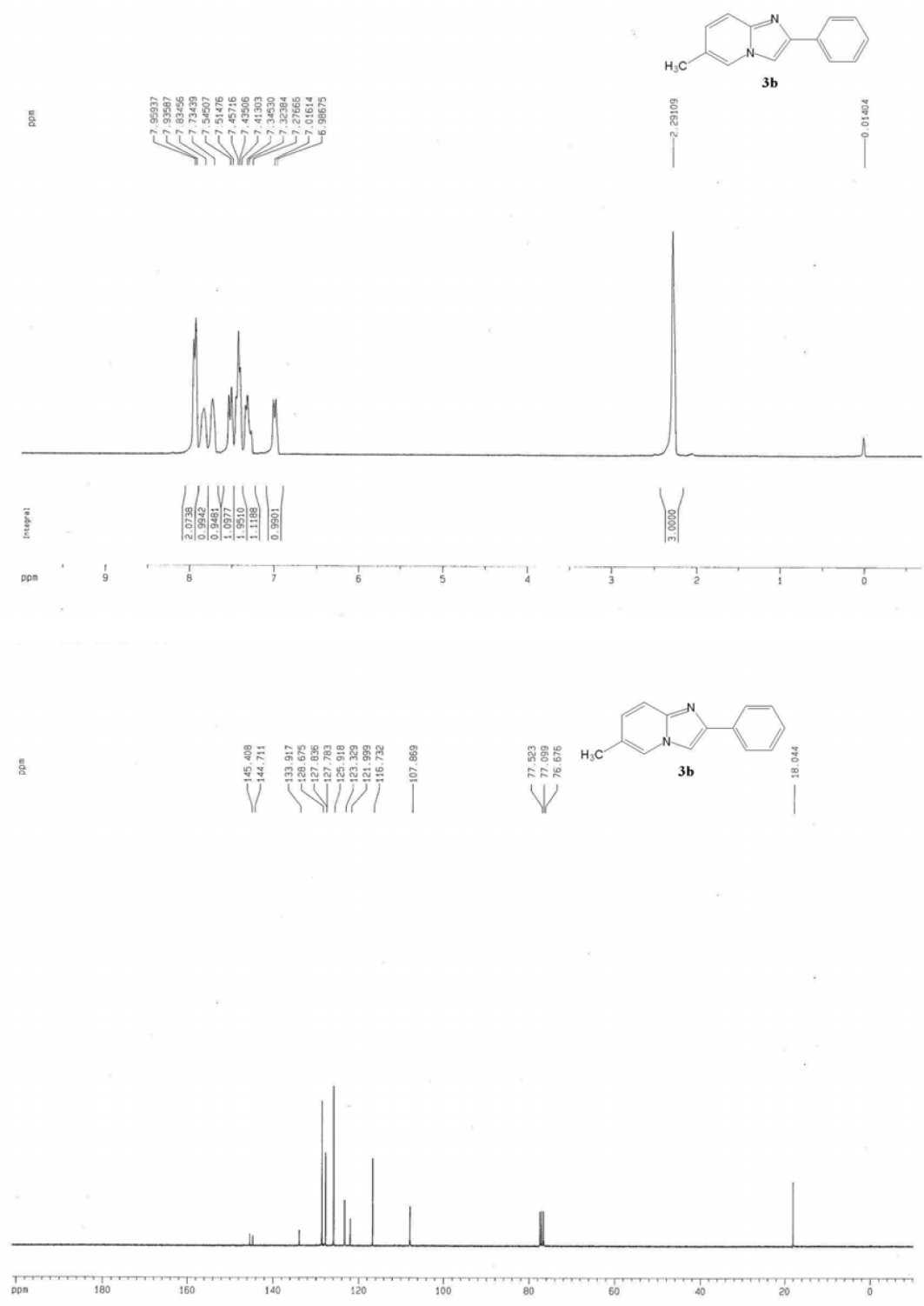

Figure S2. ${ }^{1} \mathrm{H}$ NMR of $\mathbf{3 b}\left(300 \mathrm{MHz}, \mathrm{CDCl}_{3}\right)$ and ${ }^{13} \mathrm{C} \mathrm{NMR}$ of $\mathbf{3 b}\left(75 \mathrm{MHz}, \mathrm{CDCl}_{3}\right)$. 

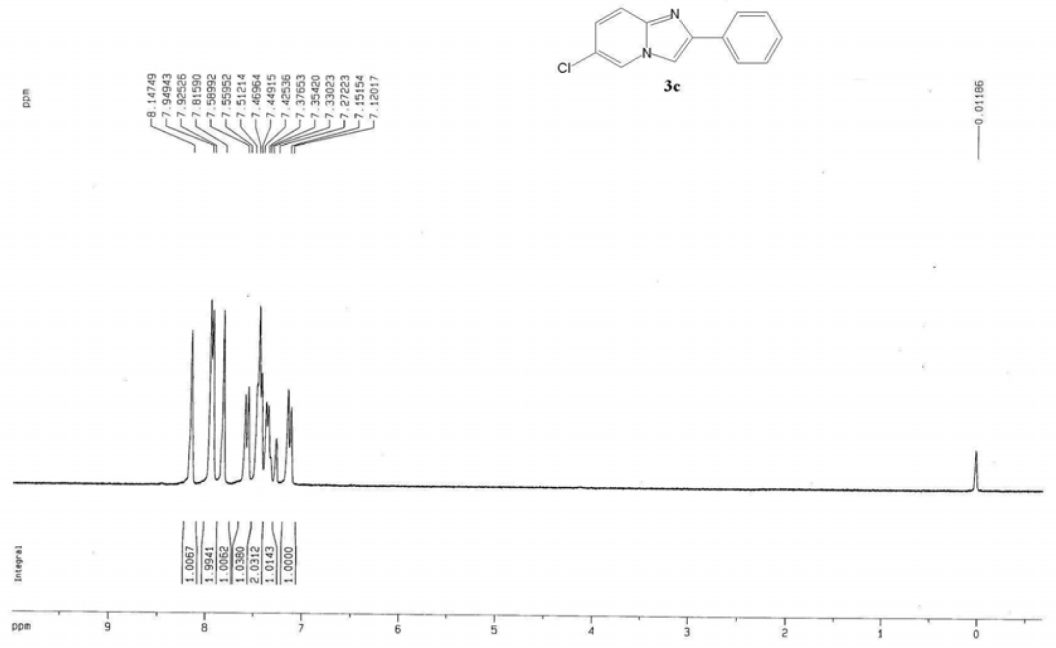

晏
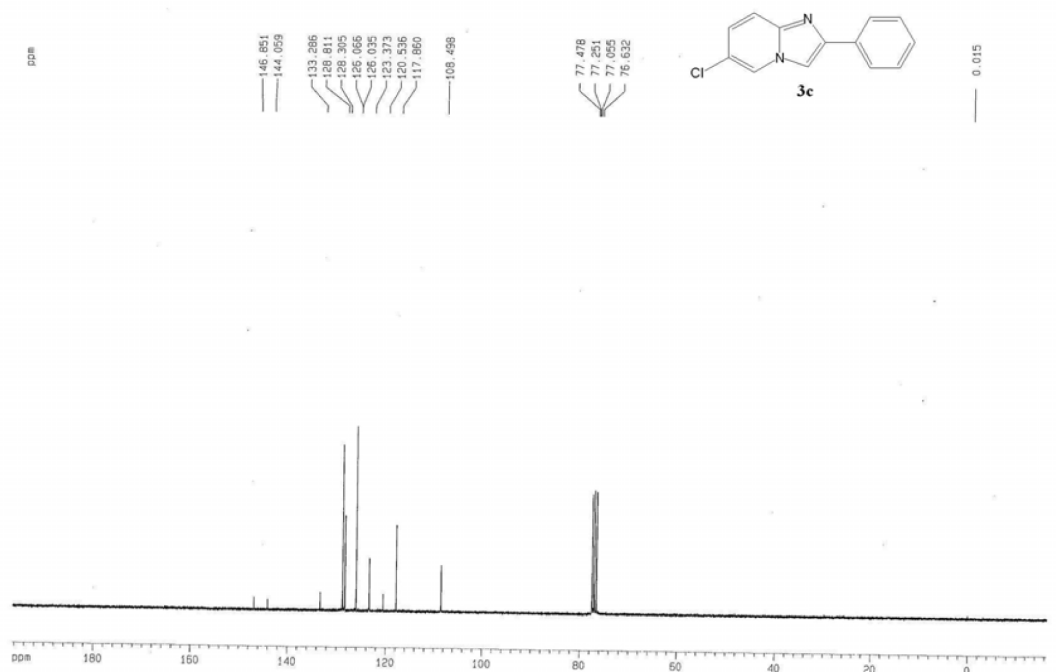

Figure S3. ${ }^{1} \mathrm{H} \mathrm{NMR}$ of $\mathbf{3 c}\left(300 \mathrm{MHz}, \mathrm{CDCl}_{3}\right)$ and ${ }^{13} \mathrm{C} \mathrm{NMR}$ of $\mathbf{3 c}\left(75 \mathrm{MHz}, \mathrm{CDCl}_{3}\right)$. 

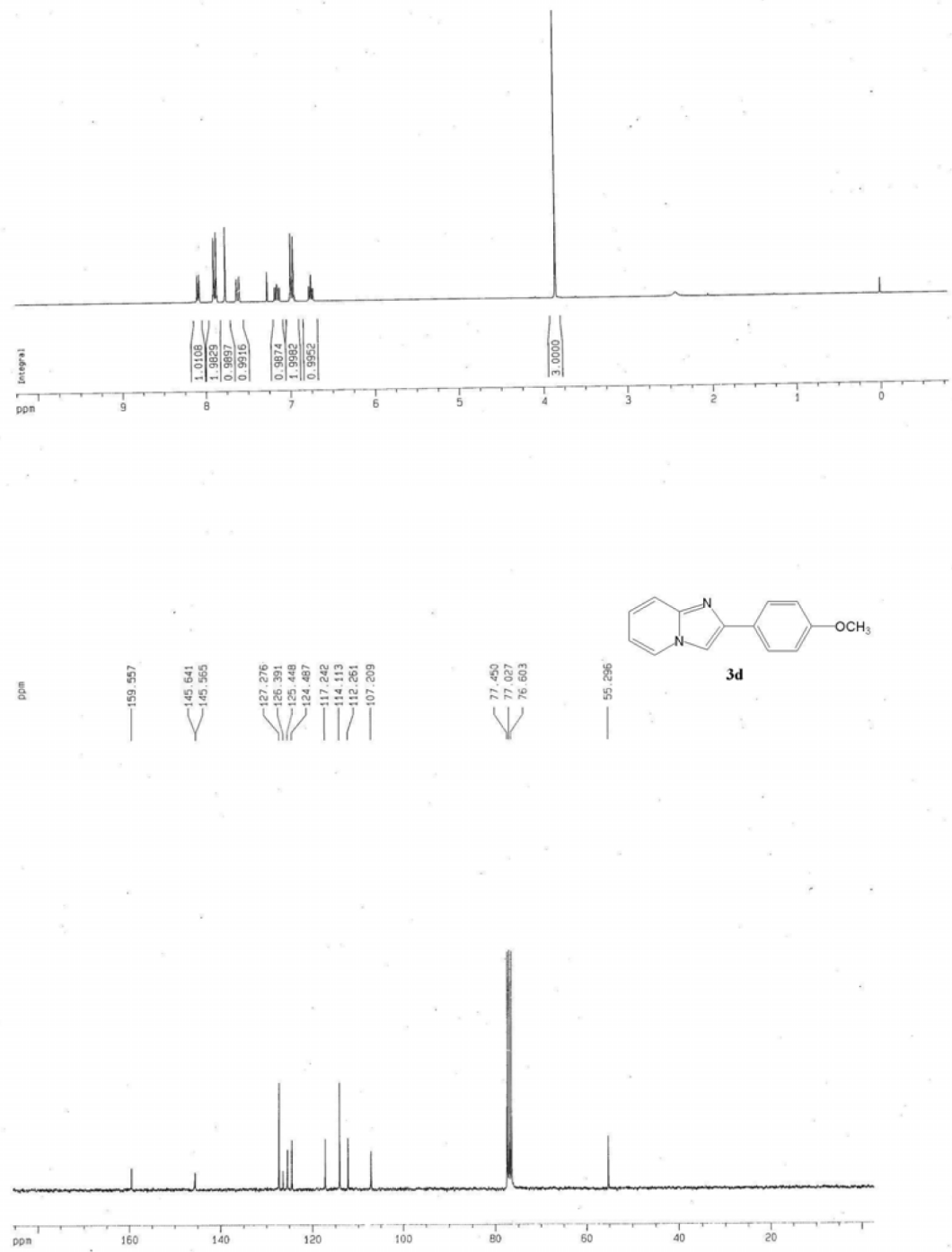

Figure S4. ${ }^{1} \mathrm{H}$ NMR of $\mathbf{3 d}\left(300 \mathrm{MHz}, \mathrm{CDCl}_{3}\right)$ and ${ }^{13} \mathrm{C} \mathrm{NMR}$ of $\mathbf{3 d}\left(75 \mathrm{MHz}, \mathrm{CDCl}_{3}\right)$. 


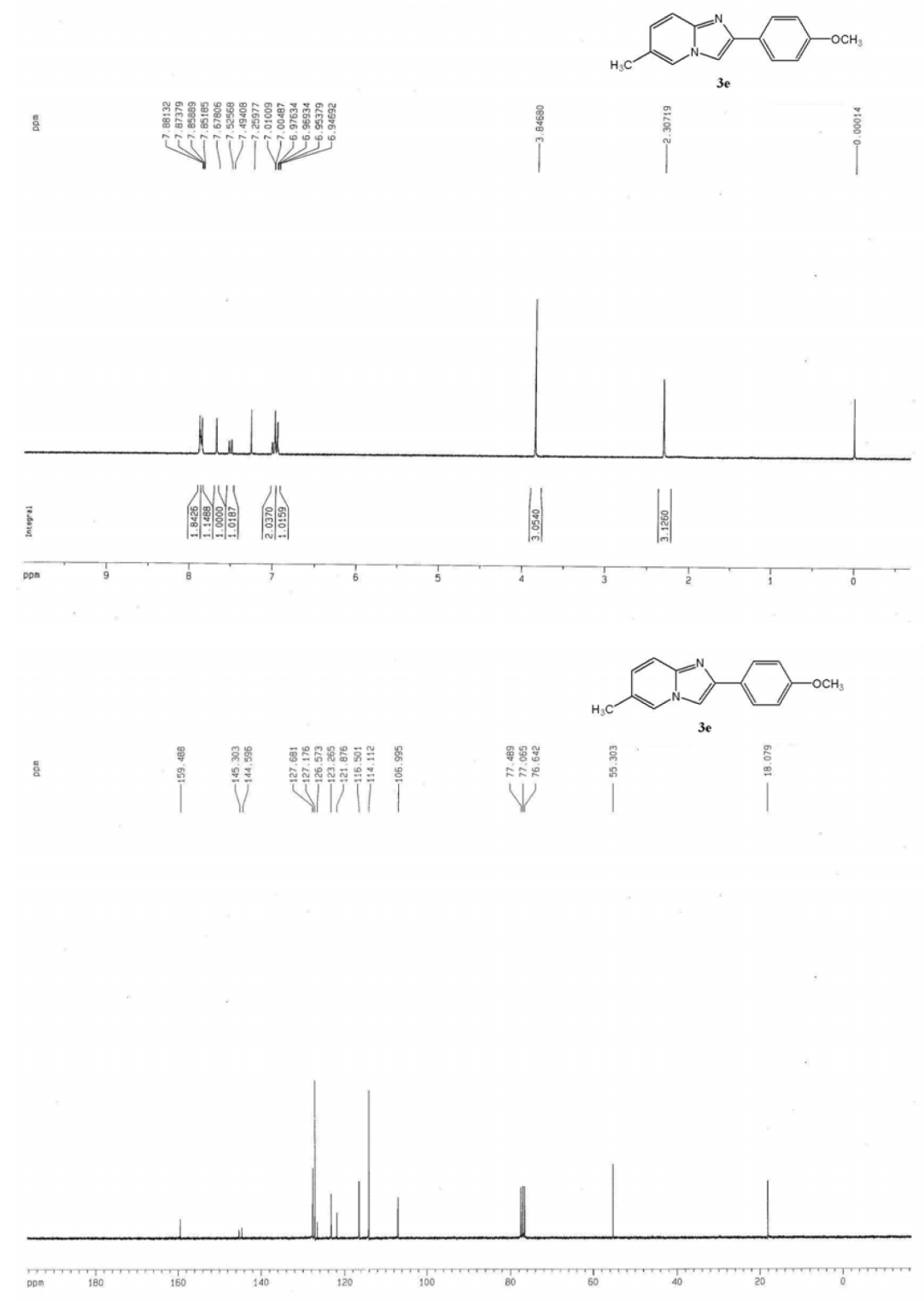

Figure S5. ${ }^{1} \mathrm{H}$ NMR of $\mathbf{3 e}\left(300 \mathrm{MHz}, \mathrm{CDCl}_{3}\right)$ and ${ }^{13} \mathrm{C} \mathrm{NMR}$ of $\mathbf{3 e}\left(75 \mathrm{MHz}, \mathrm{CDCl}_{3}\right)$. 


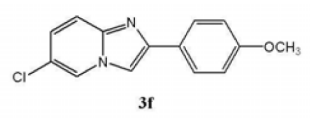

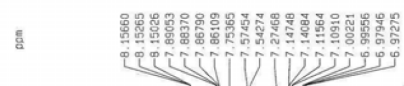
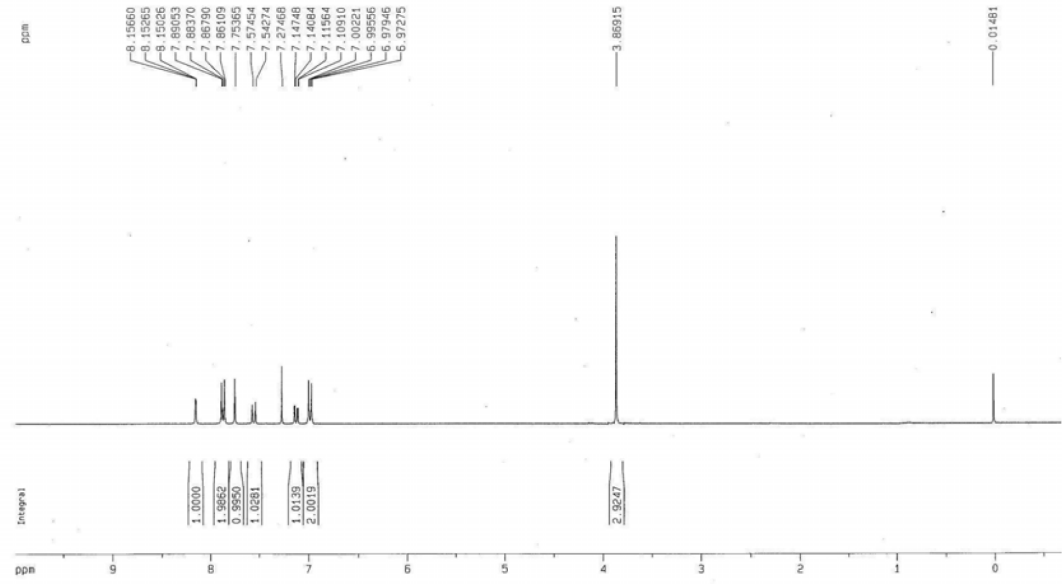

s
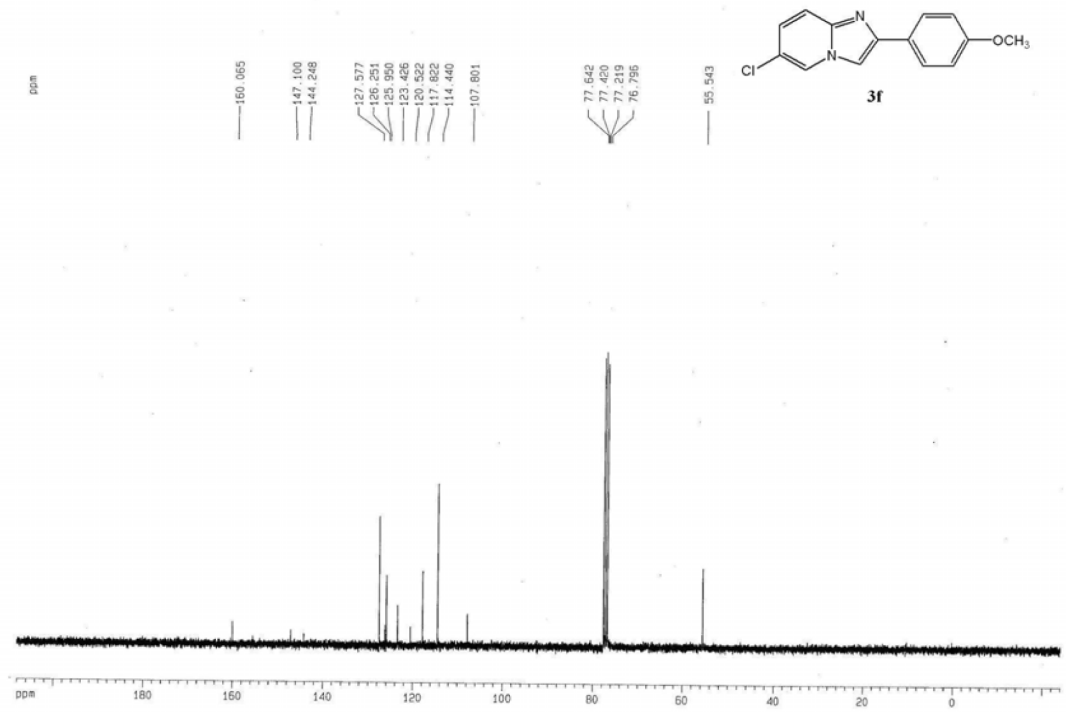

Figure S6. ${ }^{1} \mathrm{H}$ NMR of $\mathbf{3 f}\left(300 \mathrm{MHz}, \mathrm{CDCl}_{3}\right)$ and ${ }^{13} \mathrm{C} \mathrm{NMR}$ of $\mathbf{3 f}\left(75 \mathrm{MHz}, \mathrm{CDCl}_{3}\right)$. 


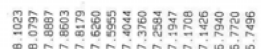

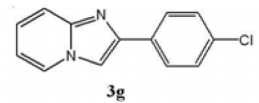

帘 نيincivivi:

$3 g$
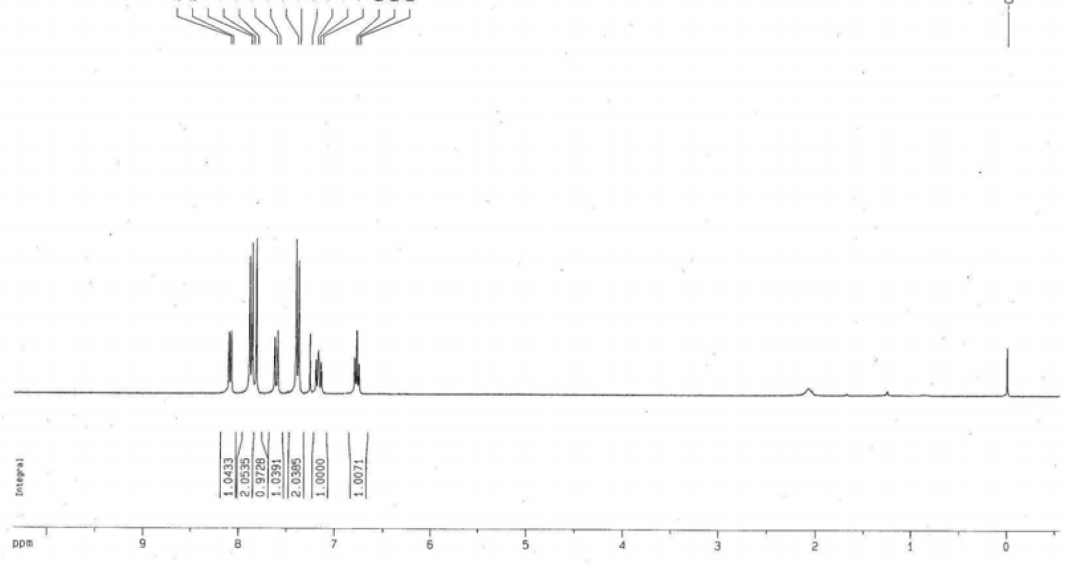

⿷
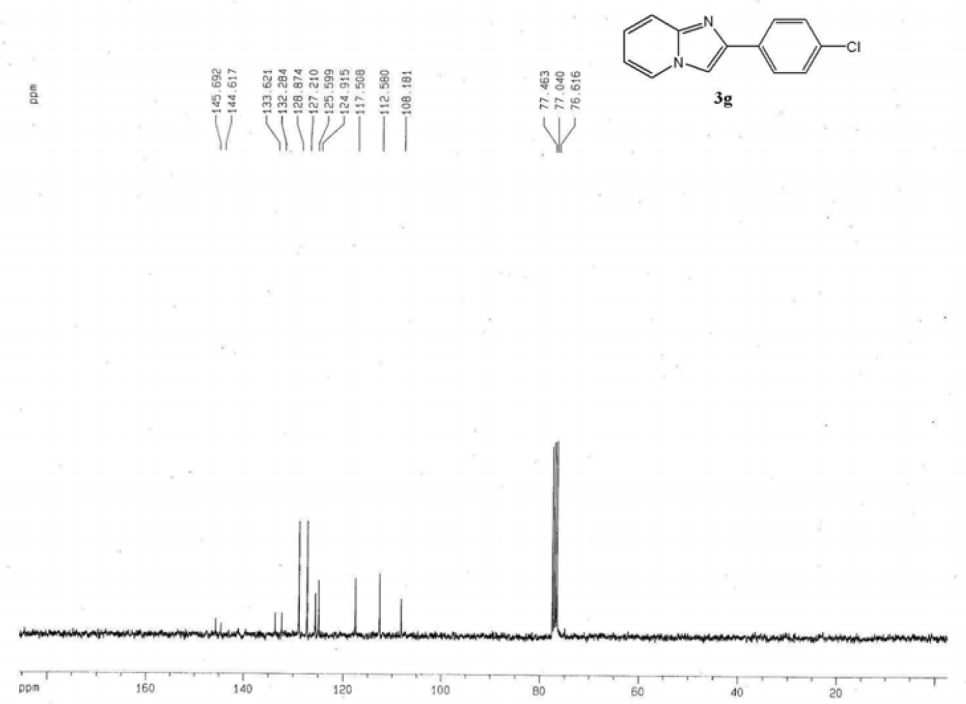

Figure S7. ${ }^{1} \mathrm{H} \mathrm{NMR}$ of $\mathbf{3 g}\left(300 \mathrm{MHz}, \mathrm{CDCl}_{3}\right)$ and ${ }^{13} \mathrm{C} \mathrm{NMR}$ of $\mathbf{3 g}\left(75 \mathrm{MHz}, \mathrm{CDCl}_{3}\right)$. 


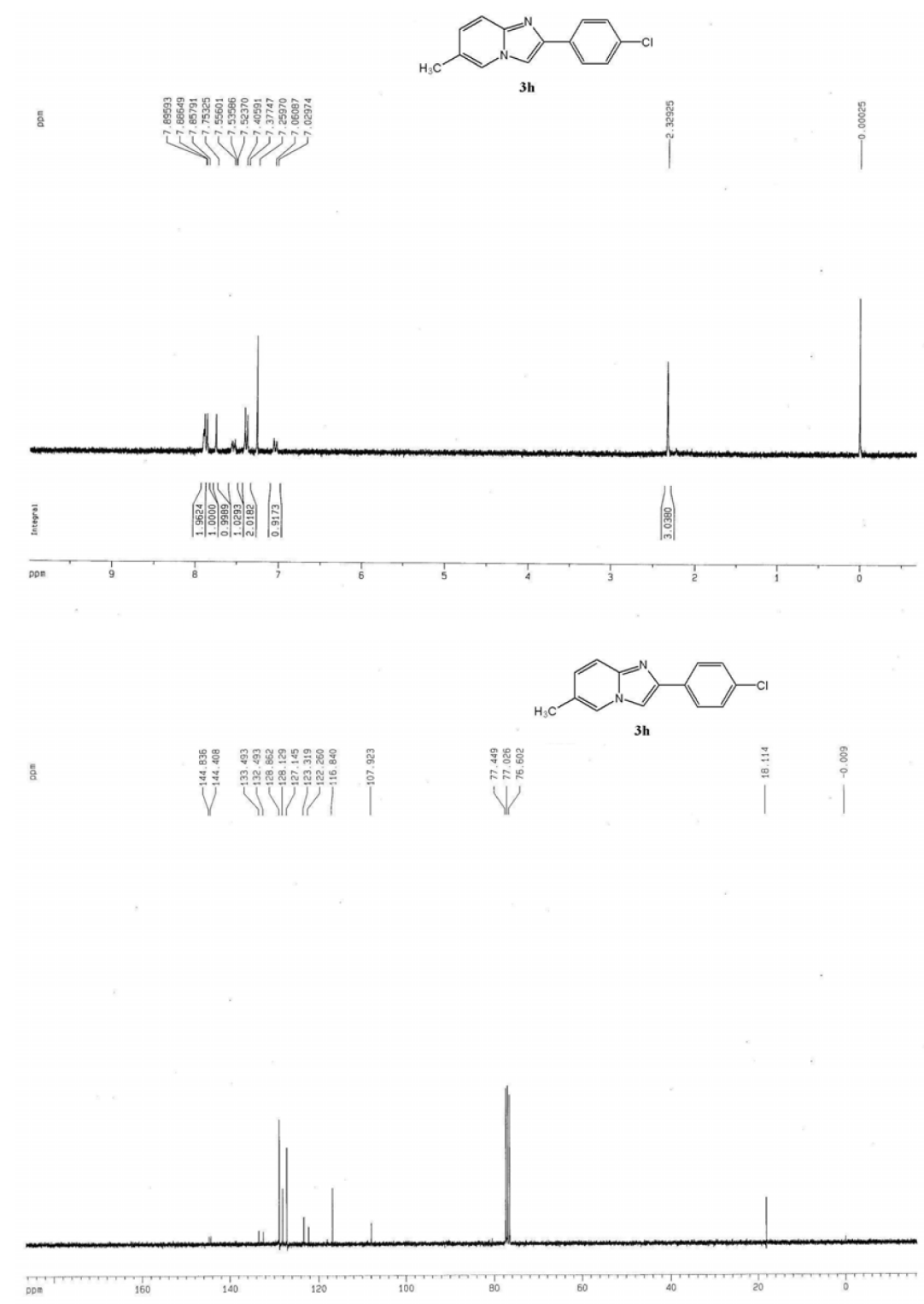

Figure S8. ${ }^{1} \mathrm{H}$ NMR of $\mathbf{3 h}\left(300 \mathrm{MHz}, \mathrm{CDCl}_{3}\right)$ and ${ }^{13} \mathrm{C} \mathrm{NMR}$ of $\mathbf{3 h}\left(75 \mathrm{MHz}, \mathrm{CDCl}_{3}\right)$. 


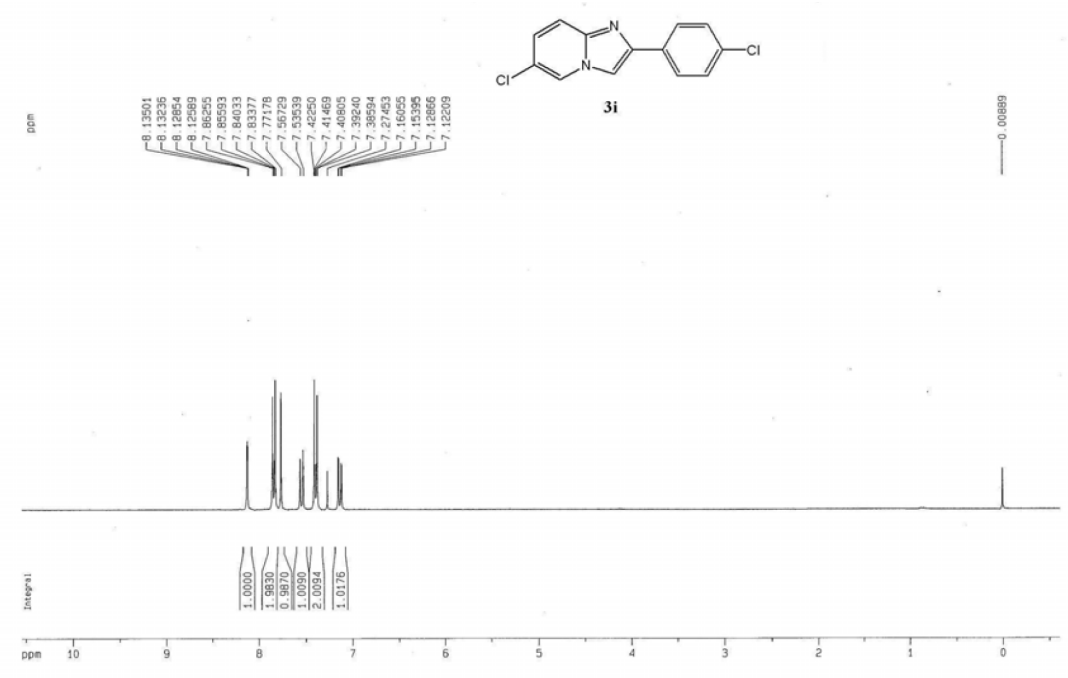

ธิ
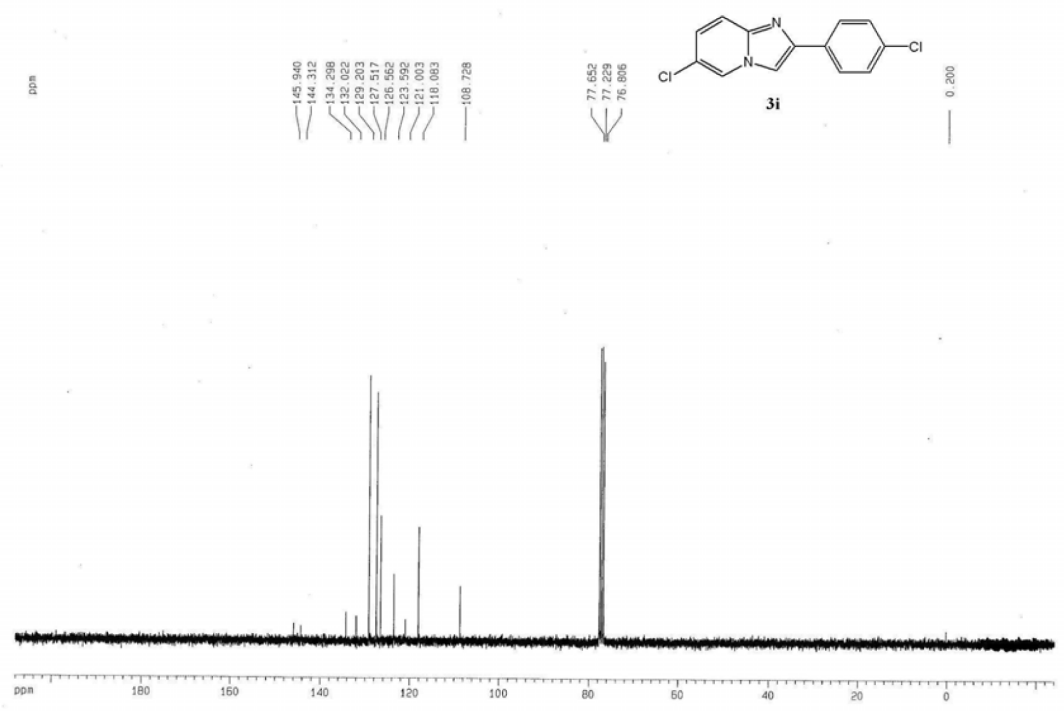

Figure S9. ${ }^{1} \mathrm{H} \mathrm{NMR}$ of $\mathbf{3 i}\left(300 \mathrm{MHz}, \mathrm{CDCl}_{3}\right)$ and ${ }^{13} \mathrm{C} \mathrm{NMR}$ of $\mathbf{3 i}\left(75 \mathrm{MHz}, \mathrm{CDCl}_{3}\right)$. 


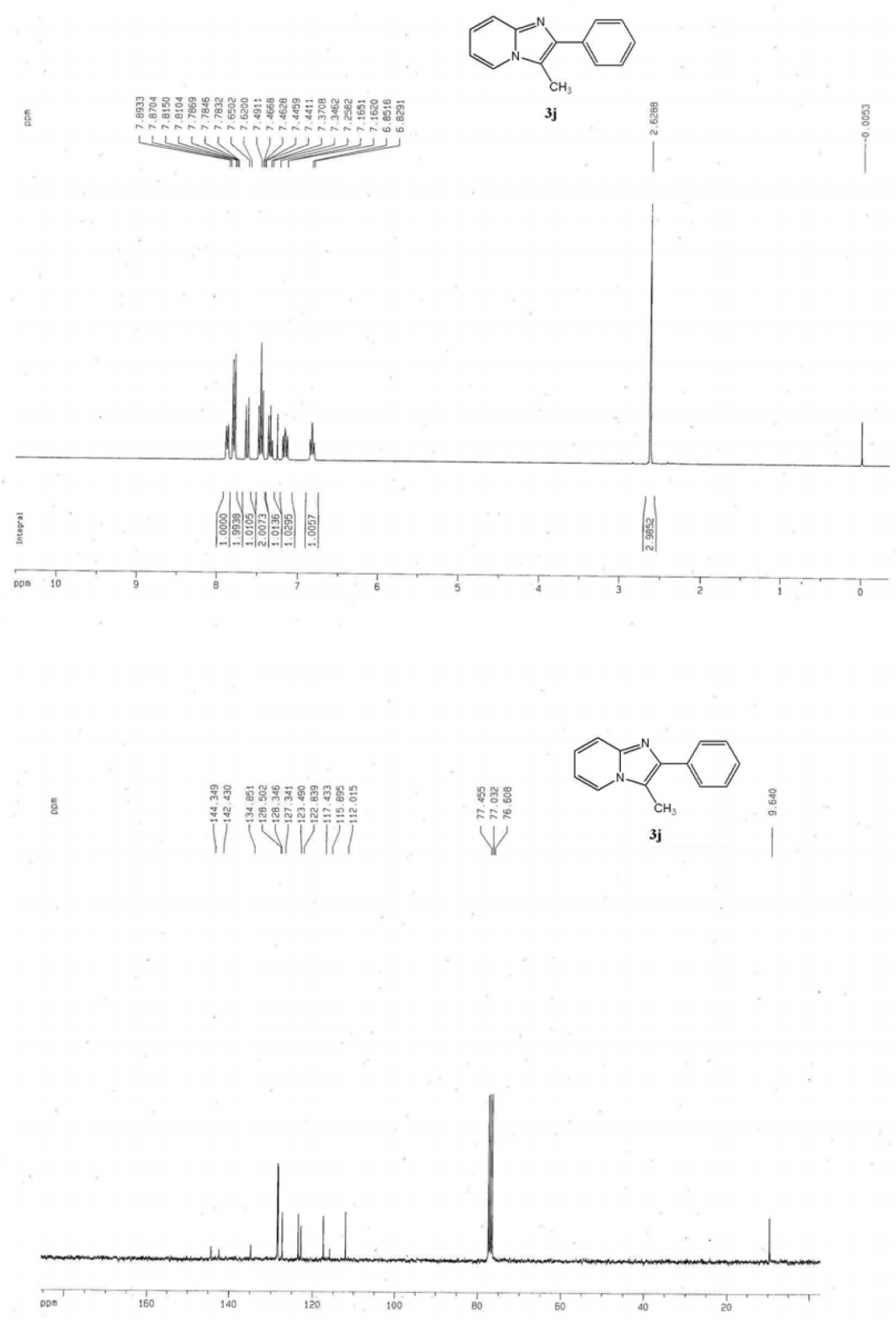

Figure S10. ${ }^{1} \mathrm{H}$ NMR of $\mathbf{3 j}$ ( $\left.300 \mathrm{MHz}, \mathrm{CDCl}_{3}\right)$ and ${ }^{13} \mathrm{C} \mathrm{NMR}$ of $\mathbf{3 j}\left(75 \mathrm{MHz}, \mathrm{CDCl}_{3}\right)$. 


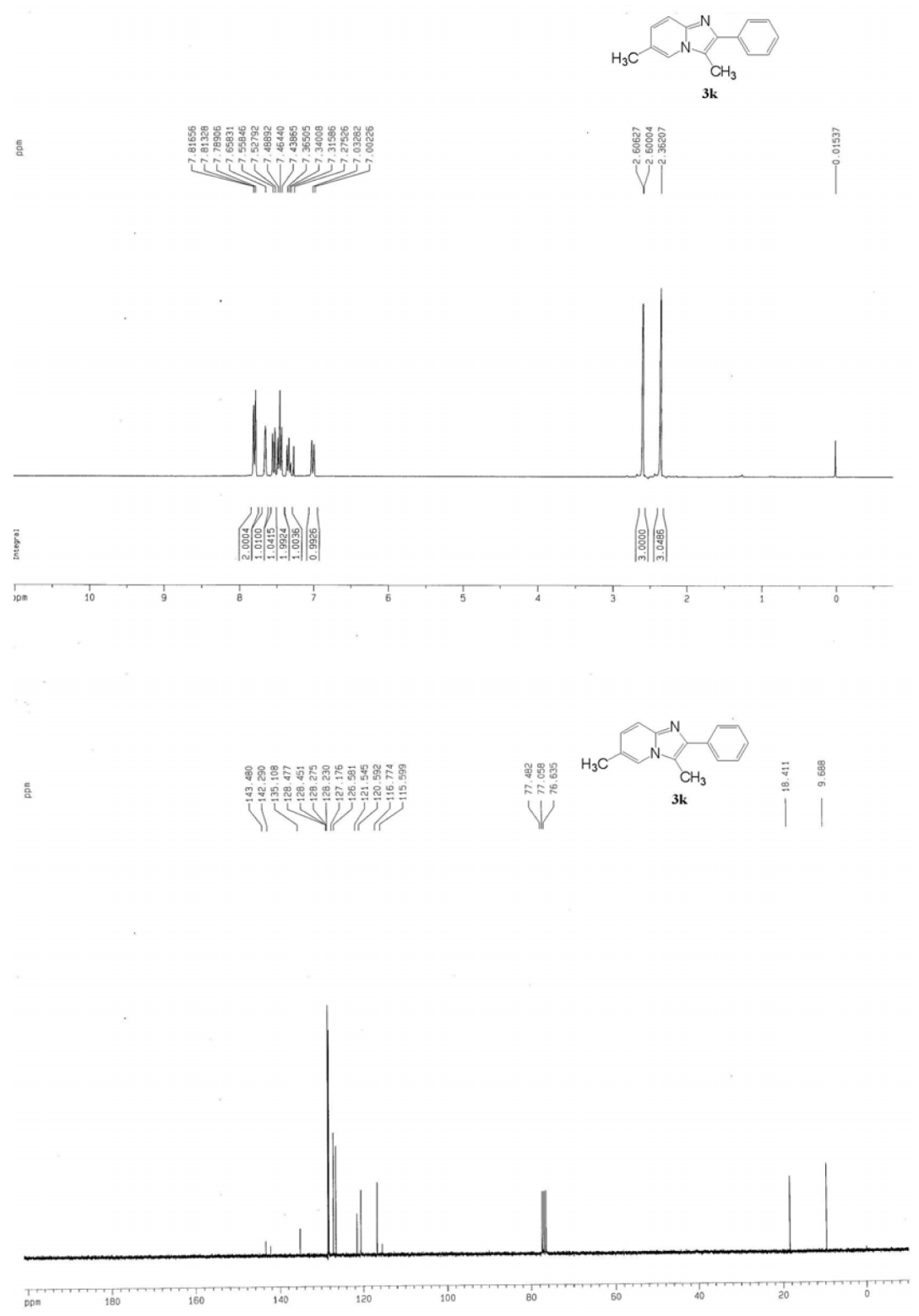

Figure S11. ${ }^{1} \mathrm{H}$ NMR of $\mathbf{3 k}\left(300 \mathrm{MHz}, \mathrm{CDCl}_{3}\right)$ and ${ }^{13} \mathrm{C} \mathrm{NMR}$ of $\mathbf{3 k}\left(75 \mathrm{MHz}, \mathrm{CDCl}_{3}\right)$. 


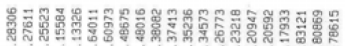

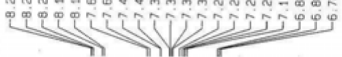

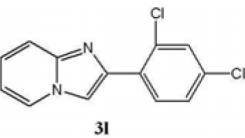

31
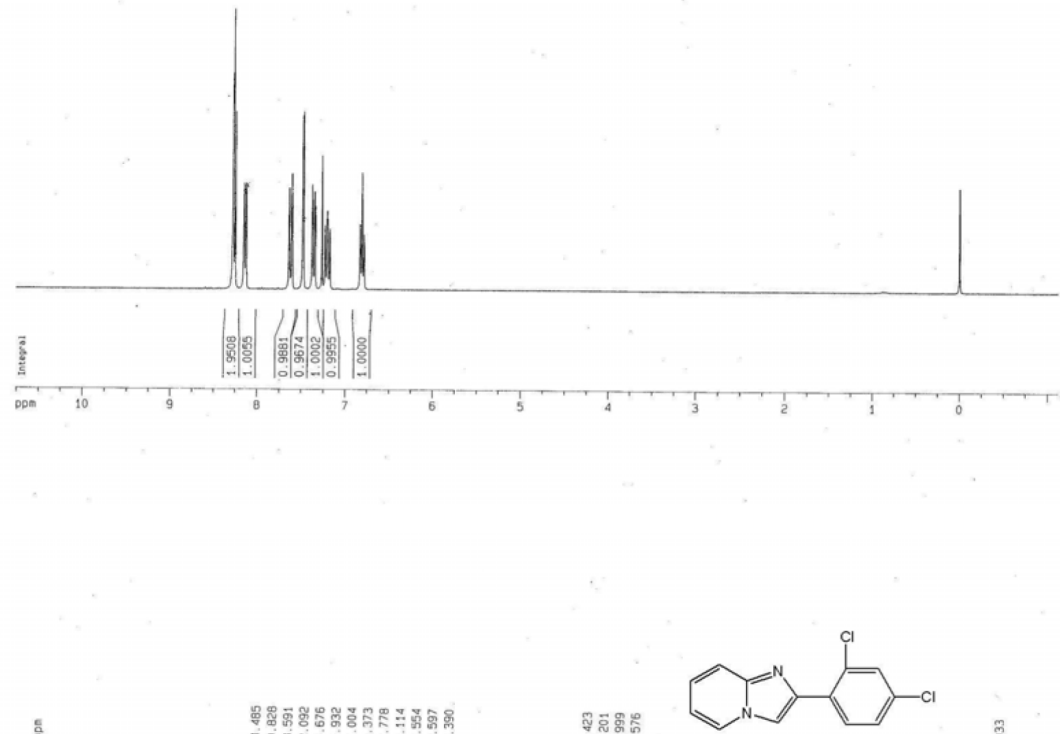

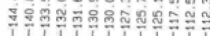

STwili

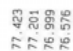

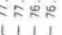

W

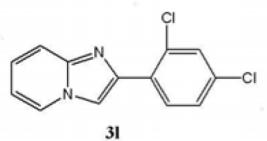

0
s.
$\vdots$
1

Figure S12. ${ }^{1} \mathrm{H} \mathrm{NMR}$ of $\mathbf{3 l}\left(300 \mathrm{MHz}, \mathrm{CDCl}_{3}\right)$ and ${ }^{13} \mathrm{C} \mathrm{NMR}$ of $\mathbf{3 l}\left(75 \mathrm{MHz}, \mathrm{CDCl}_{3}\right)$. 
Vol. 00, No. 00, 2009

Thu et al.

$\mathrm{S} 13$
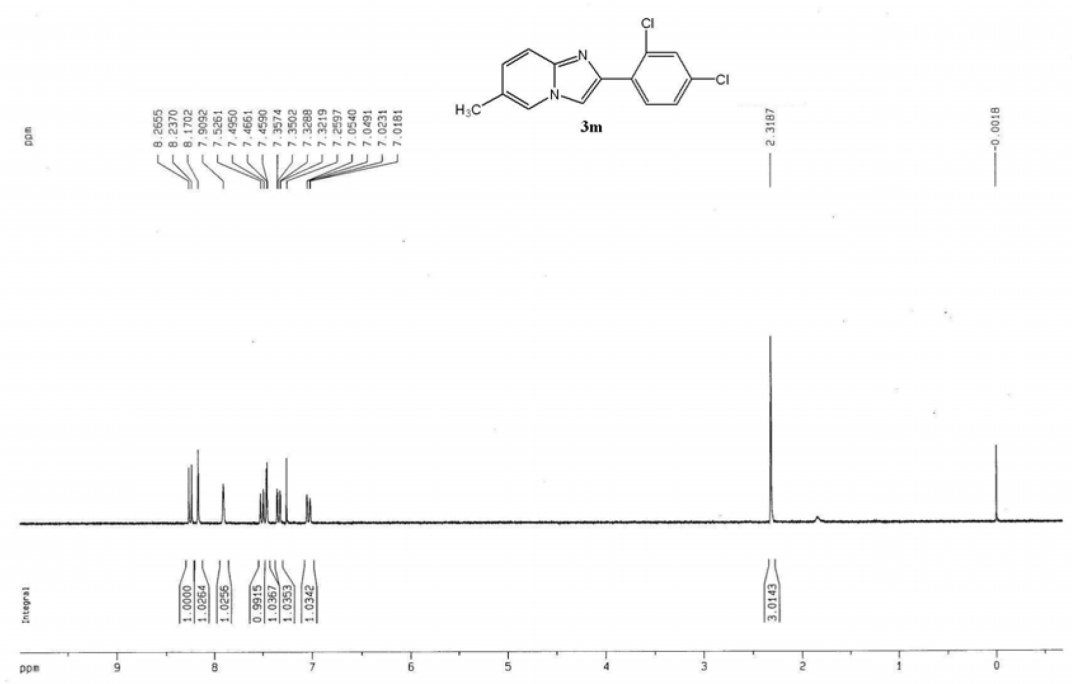

ฐ

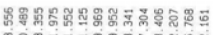
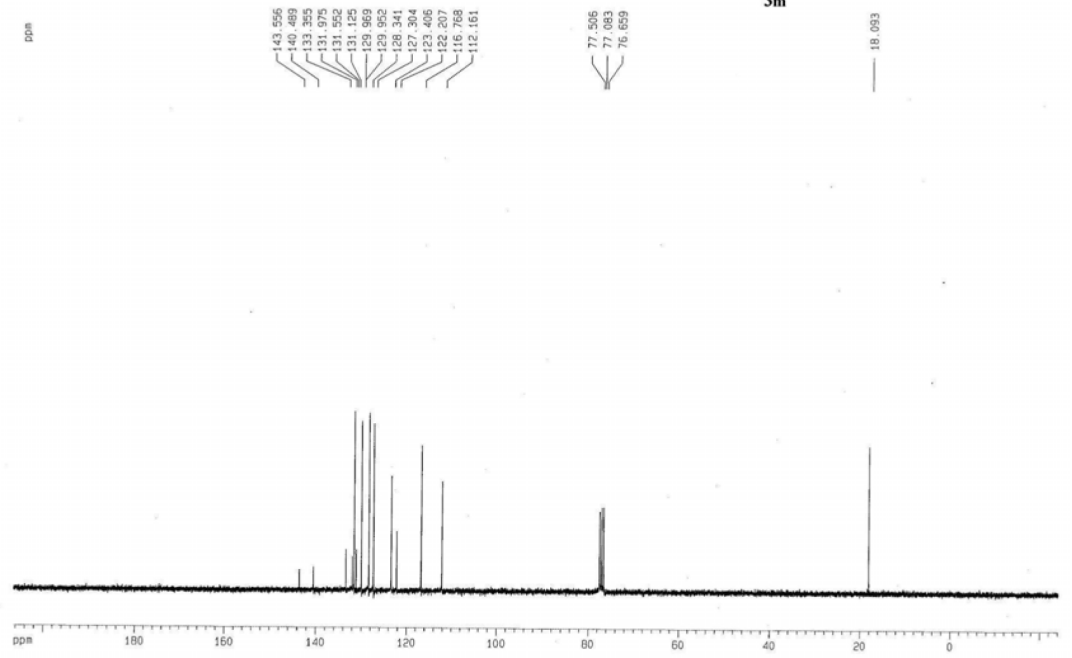

Figure S13. ${ }^{1} \mathrm{H} \mathrm{NMR}$ of $\mathbf{3 m}\left(300 \mathrm{MHz}, \mathrm{CDCl}_{3}\right)$ and ${ }^{13} \mathrm{C} \mathrm{NMR}$ of $\mathbf{3 m}\left(75 \mathrm{MHz}, \mathrm{CDCl}_{3}\right)$. 